\title{
Transport phenomena in a sidewall-moving bottom-heated cavity using heatlines
}

\author{
NIRMALENDU BISWAS and NIRMAL K MANNA* \\ Department of Mechanical Engineering, Jadavpur University, Kolkata 700 032, India \\ e-mail: nirmalkmannaju@gmail.com
}

MS received 25 September 2015; revised 13 June 2016; accepted 12 July 2016

\begin{abstract}
The understanding of basic feature of energy transport from a heat source is important from the fundamental point of view as well as from various engineering and technological applications. To enrich the knowledge in this area, this paper presents energy transport phenomena from the heated bottom of an air-filled enclosure using heatfunction and heatlines. Both upward motion and downward motion of sidewalls and the alteration of cooling between sidewalls and top wall are considered, which yields four different cases. All the cases are investigated to identify the proper combination of wall motion and thermal condition for better thermal performance, considering different convection regimes. The highly nonlinear nature of flow is solved numerically using an in-house code, taking into account different speeds of wall motion and relative strength of buoyant flow and shear flow. The results reveal that the case with side cooling and downward translation of sidewalls performs maximum heat transfer compared with other cases. Higher speed of wall translation also causes higher heat transfer. Under natural convection regime, heat transfer is significantly high. Furthermore, the order of thermal mixing in a cavity is analysed and it is found that top cooling causes higher thermal mixing. To demonstrate the vortical flow structure in the cavity, streamfunction and streamlines are used. Evolutions of symmetric and asymmetric flow vortices with centre and saddle points and energy recirculation cells are found in the cavity.
\end{abstract}

Keywords. Mixed convection; moving boundary; heatfunction; heatlines; thermal mixing.

\section{Introduction}

Understanding of transport phenomena of a bottom-heated cavity with moving sidewalls is important due to its relevance in different areas of applications like materials processing, flow and heat transfer in solar ponds, crystal growth, food processing, float glass production, galvanizing and metal coating, continuous drying, lubrication technologies, microprocessors and electronic components cooling. Complexity arises from the coupled effect of thermal heating and mechanical movement of sidewalls which controls the transport phenomena of fluid flow and heat transfer of the fluid contained in the cavity or enclosure. Literature review shows that the moving-wall cavity or driven cavity (or lid-driven cavity) has received a considerable amount of attention from many researchers from longperiod, and vast amount of work has been carried out on it. Still a good number of researches are continuing every year on different aspects [1-12] of this lid-driven cavity problem for further enrichment of knowledge in this area. Earlier, studies were conducted considering differentially heated cavity [1, 3, 13-16], irregular shape of cavity

*For correspondence wall [17], inclination of the cavity [18], and using different transport media $[19,20]$ and embedded obstacle [21-23] in the flow domain. Ramakrishna et al [19] investigated the heat flow during the mixed convection involving various thermal boundary conditions along with the adiabatic top wall that translates. A comprehensive review on the driven cavity has been reported by Shankar and Deshpande [24]. Lid-driven cavity geometry is also used as a benchmark configuration for the numerical solution of Navier-Stokes equations [25].

From the previous studies on thermal convection in liddriven cavities, it is observed that the complexity in heat and fluid flow characteristics increases when direction of the wall movement changes [13-16], and also when number of moving walls changes from one lid [26] to two [15] or more [16] lids. Recently, Roy et al [8] investigated the effects of a single-lid motion and two-lid motion of a sidecooled, top adiabatic and isothermally bottom-heated, square enclosure. This study has indicated that the direction of lid motion strongly influences the thermal convection heat transfer.

Several recent studies have been conducted on lid-driven cavity considering thermal convection generated from uniformly heated bottom wall [27-31]. Moallemi and Jang 
[29] investigated the fluid flow and heat transfer characteristics considering sidewalls as adiabatic, and top cold wall as moving. Transport mechanism of laminar mixed convection in a shear- and buoyancy-driven cavity with moving cooled sidewalls has been investigated by Aydin and Yang [27]. Waheed [31] performed numerical investigation for mixed convection in a rectangular enclosure, considering thermally insulated right vertical wall and other cold walls. Studies involving isothermally heated bottom wall with moving top cold wall $[28,30]$ were also reported. The numerical investigation of mixed convection heat transfer in a three-dimensional cavity with the moving top wall was studied by Seyyedi et al [32]. They found that sliding angles have significant effect on heat transfer enhancement.

The investigation on the driven cavity has also been extended considering the heated top wall that moves and cooled stationary bottom wall [33, 34], and bottom-heated inclined cavity filled with nanofluid and cooled from sidewalls [35], partially heated from bottom and/or sidewall [36-38]. Muthtamilselvan and Doh [39] investigated uniform and non-uniform heating of bottom wall on mixed convection in a lid-driven square cavity filled with nanofluid.

Literature survey also shows that except for a few recent works $[8,19,26]$, most of the earlier works had utilized streamlines and isotherms to depict transport phenomenon in the cavity. However, isotherm plots are most appropriate tool for visualizing heat transport when the heat transfer is governed by only conduction. In the presence of convection, heatfunction and heatlines can properly explain the transport phenomenon of energy flow in the cavity. Present work is dedicated to elucidate the energy flow dynamics using heatfunction and heatlines (along with usual isotherms, streamlines and Nusselt number). The concept of heatfunction and heatlines was developed from steady-state energy balance equation by Kimura and Bejan [40] and latter, further work was done on it by Costa [41].

Lid-driven cavity is well-known classical type of problem. This type of problem is specifically suited for production of float glass [42], chemical processing, lubrication technologies [43], galvanizing, metal casting, coaters for producing high-grade paper and photographic film and many others [24].

Basic understanding of the effect of thermal boundary condition of sidewalls' moving and bottom-heated cavity under buoyant flow situation is important on the purview of different multi-physical applications that couple with one or more areas of porous-media, heat generation, debris cooling in nuclear application, chemical reaction, nanofluids, magnatohydrodynamics, etc. [44-52]. Literature review indicates that the study on bottom-heated cavity with moving sidewalls under different thermal boundary conditions is found to be limited. Under bottom-heated configuration, different thermal conditions of other walls along with the motion of sidewalls are studied systematically in this work. The aim of the present study is to explore comprehensive understanding of transport phenomena of energy flow using heatlines (in conjunction with the fluid flow using streamlines), to analyse heat transfer characteristics of the bottom-heated cavity using Nusselt number and to identify the proper combination of lid motion and thermal condition for the better thermal performance. All the studies are carried out using air $(\mathrm{Pr}=0.71)$ and considering different flow regimes such as forced, mixed and natural convection through Richardson number (Ri) ranging from 0.01 to 100 .

\section{Physical description and mathematical formulation}

The geometry of bottom-heated cavity with moving sidewalls is shown in figure 1. It consists of two-dimensional upright square cavity of side $L$ filled with air, where both the sidewalls are translating at a constant speed, $v_{w}$ in their own planes either vertically upward or vertically downward. The coupling of thermally induced flow (due to differential heating) with shear-induced flow (due to mechanical motion of sidewalls) governs the heat and fluid flow in the cavity. To understand their combined effect on the heat and fluid flow, four different configurations (Cases 1 to 4 ) are considered for investigation by interchanging the isothermal and adiabatic conditions between the sidewalls and the top wall for both upward and downward motion of sidewalls. For all the cases, the heated bottom wall is assumed as maintaining a constant temperature $T_{h}$. The sidewalls (for cases 1 and 3 ) or the top wall (for cases 2 and 4) is at a lower temperature $T_{c}$, which depends on the temperature of surrounding ambient.

Conservation principles for mass, momentum and energy are utilized to formulate the required governing equations in Cartesian coordinate system with the origin attached to the lower left corner of the cavity. Assuming a steady incompressible laminar fluid flow within the limits of Boussinesq approximation, the governing equations are expressed in non-dimensional forms as

$$
\begin{gathered}
\frac{\partial U}{\partial X}+\frac{\partial V}{\partial Y}=0 \\
U \frac{\partial U}{\partial X}+V \frac{\partial U}{\partial Y}=-\frac{\partial P}{\partial X}+\frac{1}{\operatorname{Re}}\left(\frac{\partial^{2} U}{\partial X^{2}}+\frac{\partial^{2} U}{\partial Y^{2}}\right) \\
U \frac{\partial V}{\partial X}+V \frac{\partial V}{\partial Y}=-\frac{\partial P}{\partial Y}+\frac{1}{\operatorname{Re}}\left(\frac{\partial^{2} V}{\partial X^{2}}+\frac{\partial^{2} V}{\partial Y^{2}}\right)+\operatorname{Ri} \theta \\
U \frac{\partial \theta}{\partial X}+V \frac{\partial \theta}{\partial Y}=\frac{1}{\operatorname{Re} \operatorname{Pr}}\left(\frac{\partial^{2} \theta}{\partial X^{2}}+\frac{\partial^{2} \theta}{\partial Y^{2}}\right)
\end{gathered}
$$

where $X$ and $Y$ are the dimensionless coordinates; $U$ and $V$ are dimensionless $X$ - and $Y$-components of velocity. $\theta$ and 
Case 1 and 2

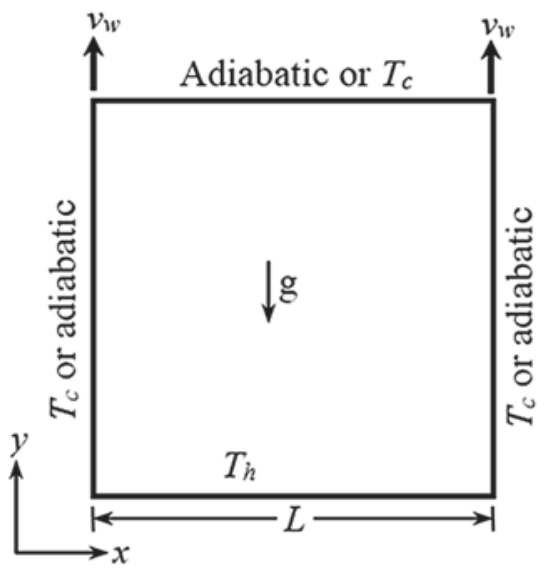

Case 3 and 4

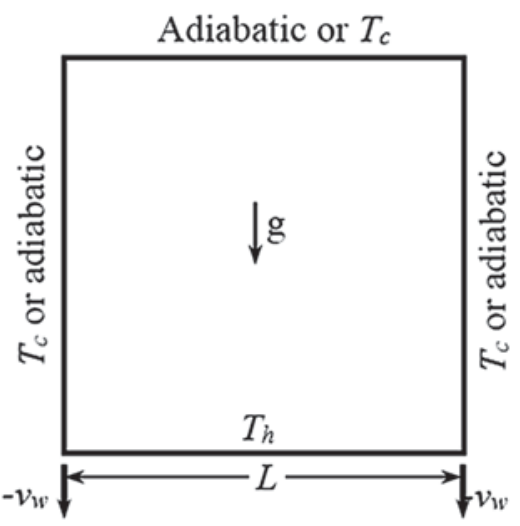

Figure 1. Schematic arrangement of the computational domain with different velocity and boundary conditions.

$P$ are dimensionless temperature and pressure, respectively. $\mathrm{Re}$, Pr and Ri are, respectively, Reynolds, Prandtl and Richardson numbers. These quantities are defined as

$$
\begin{aligned}
& (X, Y)=\frac{(x, y)}{L}, \quad(U, V)=\frac{(u, v)}{v_{w}}, \quad P=\frac{(p+\rho g y)-p_{a}}{\rho v_{w}^{2}}, \\
& \theta=\frac{T-T_{c}}{T_{h}-T_{c}}
\end{aligned}
$$

$$
\operatorname{Re}=\frac{v_{w} L}{v}, \quad \operatorname{Pr}=\frac{v}{\alpha}, \quad \operatorname{Ri}=\frac{\mathrm{Gr}}{\operatorname{Re}^{2}}, \quad \mathrm{Gr}=\frac{g \beta\left(T_{h}-T_{c}\right) L^{3}}{v^{2}}
$$

where $T$ and $p$ are the dimensional temperature and pressure; $u$ and $v$ are dimensional velocity components along the $x$ and $y$ axes. Different transport properties of fluid such as density $\rho$, kinematic viscosity $v$, volumetric thermal expansion coefficient $\beta$, and thermal diffusivity $\alpha$ are assumed to be constant except the density in the body force term that varies with temperature following the Boussinesq approximation [53]. Viscous dissipation is neglected during energy balance in Eq. (3) as it contributed insignificantly $[54,55]$.

The boundary conditions for the governing equations (1)-(3) are set as follows:
The positive/negative sign before $V$ denotes upward/downward motion of the sidewalls.

As a part of post-processing, the heatfunction and streamfunction, and the Nusselt number are computed, and these are utilized to analyse the energy and fluid flow, and the heat transfer characteristics for the present problem. The concept of heatfunction $(\Pi)[8,26,40,41,56,57]$ can help properly appreciating the steady-state energy flow of working fluid in the cavity. The heatfunction intrinsically satisfies the steady-state energy balance equation, and consists of both conductive $(-(\partial \theta / \partial X),-(\partial \theta / \partial Y))$ and convective $(U \theta, V \theta)$ heat fluxes. For the present work, it is expressed in the first-order differential form as

$$
-\frac{\partial \Pi}{\partial X}=V \theta-\frac{1}{\operatorname{Re} \operatorname{Pr}} \frac{\partial \theta}{\partial Y} \quad \text { and } \quad \frac{\partial \Pi}{\partial Y}=U \theta-\frac{1}{\operatorname{Re} \operatorname{Pr}} \frac{\partial \theta}{\partial X}
$$

and solved iteratively using an integration method, applying Dirichlet and Neumann boundary conditions on temperature, respectively, for adiabatic and isothermal walls, and setting a reference value of $\Pi=0$ at $X=0.5, Y=0$. The constant heatfunction lines or heatlines are plotted for the visualization of heat transport from the heat source to the heat sink. On the other hand, the overall fluid-flow pattern in the cavity is interpreted through the streamfunction $(\Psi)$, which is defined as

on bottom wall $(Y=0$ and $0 \leq X \leq 1), U=V=0, \theta=1$, on vertical side walls $(X=0,1$ and $0 \leq Y \leq 1), U=0, \theta=0$ or $\partial \theta / \partial X=0, V= \pm 1$, and on top wall $(Y=1$ and $0 \leq X \leq 1), U=V=0, \partial \theta / \partial Y=0$, or $\theta=0$. 


$$
-\frac{\partial \psi}{\partial X}=V \quad \text { and } \quad \frac{\partial \psi}{\partial Y}=U
$$

It is also solved following the integration method by setting $\Psi=0$ on all enclosure's walls as these walls are impermeable. The clockwise (CW) and counterclockwise (CCW) rotations of fluid or energy are indicated by the negative and positive signs, respectively.

The rate of heat transfer from the heated bottom wall of the cavity are expressed in terms of local and average Nusselt number, respectively, as

$$
\mathrm{Nu}(X)=-\left.\frac{\partial \theta}{\partial Y}\right|_{Y=0} \text { and } \quad \mathrm{Nu}_{\mathrm{avg}}=\int_{0}^{1} \mathrm{Nu}(X) d X .
$$

\section{Numerical procedure and code validation}

The governing equations, Eqs. (1)-(3), along with boundary conditions, Eq. (5), are solved iteratively using an in-house CFD code based on the finite volume method (FVM) along with staggered grid system, utilizing SIMPLE algorithm [58], tri-diagonal matrix algorithm (TDMA) and alternating direction implicit (ADI) sweep. The iterative process continues until it fulfils the convergence criteria, which are set by the values of the maximum residuals and mass-defect less than $10^{-6}$ and $10^{-8}$, respectively.

A detailed discussion on the numerical procedure along with validation of this code has been reported in an earlier work [59, 60]. Furthermore, an air-filled, bottom-cooled square cavity with heated moving lid at the top as reported in $[8,31,61,62]$ is simulated using $\operatorname{Pr}=0.71$ and $\mathrm{Gr}=100$, and corresponding results for the average Nusselt number $\left(\mathrm{Nu}_{\mathrm{avg}}\right)$ at top wall are given in table 1 . The present results are compared against the published results and found in good agreement.

For the selection of appropriate grid size for the present problem, a grid independence study using $\mathrm{Nu}_{\mathrm{avg}}$ at the bottom hot wall is conducted using four different grid sizes $(40 \times 40,60 \times 60,80 \times 80,100 \times 100$ and $120 \times 120)$ for Case 1 at $\mathrm{Re}=200$ and various $\mathrm{Ri}$ values $(\mathrm{Ri}=0.1-100)$. For each grid size, the number of grids in
$X$ and $Y$ directions is taken to be the same. The grids are distributed non-uniformly, with the finer grids near the walls, so that the boundary layer can be resolved properly. The grid-study results are shown in table 2, which indicate that the percentage error (shown within bracket) with respect to the immediate coarser grid reduces sufficiently $(<1.3 \%)$ after the grid size $80 \times 80$. As such, $100 \times 100$ grid size is chosen for the present numerical computation with reasonable computational speed.

\section{Results and discussion}

The present study aims at assessing the effects of various influencing factors like the direction of lids' motion and its speed (by $\operatorname{Re}=10-200$ ), relative strength of thermally induced flow over shear flow (by $\mathrm{Ri}=0.01-100$ ) and position of cold wall on the heat transfer and thermal-fluid flow structure. Details of the study plan and the values of parameters are mentioned in table 3. The ranges of Richardson numbers $(\mathrm{Ri}=0.01-100)$ and Reynolds numbers $(\mathrm{Re}=10-200)$ correspond to Grashof numbers $(\mathrm{Gr})$ ranging from 1 to $4.0 \times 10^{6}$. At low $\mathrm{Re}$ and high $\mathrm{Ri}$, the natural convection mainly controls the heat and low fluid dynamics and the corresponding flow pattern is almost independent of the direction of lid motion. On the other hand, at high Re with low Ri, the flow dynamics is dominated by forced convection. Visualization of energy transport in the cavity is carried out using heatlines, whereas fluid flow is by streamlines. Heat transfer is computed in terms of average Nusselt number $\left(\mathrm{Nu}_{\mathrm{avg}}\right)$ of the heated bottom wall.

Circulation of confined fluid in a bottom-heated cavity with all stationary walls presents different vortical patterns depending upon the thermal condition of the top wall and the sidewalls of the cavity as shown in figure 2 . When the sidewalls are cold and the top wall is adiabatic, the fluid from bottom region of the cavity rises up to about the midvertical plane like hot plume and generates two symmetric circulations irrespective of $\mathrm{Gr}$ values as shown in figure $2 \mathrm{a}$. When the thermal conditions are interchanged (i.e., sidewall adiabatic and top wall cold), the flow pattern in the cavity changes from two circulations to single circulation depending upon $\mathrm{Gr}$ value as shown in figure $2 b$. These heat

Table 1. Comparisons of the present and the published results $[8,31,61,62]$ for average Nusselt number $\left(\mathrm{Nu}_{\mathrm{avg}}\right)$ at $\mathrm{Pr}=0.71$ and $\mathrm{Gr}=100$.

\begin{tabular}{lccccc}
\hline Re & Present results & Roy et al [8] & Waheed [31] & Iwatsu [62] & Abdelkhalek [61] \\
\hline 1 & 1.000 & 0.998 & 1.00033 & - & 1.94 \\
100 & 1.975 & 1.967 & 2.03116 & 3.84 & 1.9850 \\
400 & 3.995 & 4.004 & 4.02462 & - & 3.8785 \\
500 & 4.522 & 4.509 & 4.52671 & 6.33 & - \\
1000 & 6.431 & 6.443 & 6.48423 & 6.3450 \\
\hline
\end{tabular}


Table 2. Grid independence study using the average Nusselt number $\left(\mathrm{Nu}_{\mathrm{avg}}\right)$ at bottom wall.

\begin{tabular}{lccccc}
\hline $\mathrm{Ri}$ & $40 \times 40$ & $60 \times 60$ & $80 \times 80$ & $100 \times 100$ & $120 \times 120$ \\
\hline 0.01 & 10.308 & $10.519(2.05 \%)$ & $10.645(1.20 \%)$ & $10.771(1.18 \%)$ & $10.869(0.91 \%)$ \\
0.1 & 10.326 & $10.539(2.07 \%)$ & $10.667(1.21 \%)$ & $10.794(1.19 \%)$ & $10.919(1.16 \%)$ \\
1 & 10.546 & $10.784(2.26 \%)$ & $10.926(1.31 \%)$ & $11.059(1.22 \%)$ & $11.029(0.28 \%)$ \\
10 & 12.389 & $12.568(1.44 \%)$ & $12.661(0.74 \%)$ & $12.774(0.89 \%)$ & $12.894(0.94 \%)$ \\
100 & 19.705 & $19.991(1.45 \%)$ & $20.136(0.72 \%)$ & $20.262(0.63 \%)$ & $20.364(0.50 \%)$ \\
\hline
\end{tabular}

Table 3. Study plan and the ranges of parameters.

\begin{tabular}{lccc}
\hline & Description & Re & Ri \\
\hline Case 1 & Both sidewalls cold and moving upwards & 10 & \\
Case 2 & Both sidewalls adiabatic and moving upwards & 50 & $0.01,0.1$, \\
Case 3 & Both sidewalls cold and moving downwards & 100 & $1,10,100$ \\
Case 4 & Both sidewalls adiabatic and moving downwards & 200 & \\
\hline
\end{tabular}

and fluid flow structures (under pure thermally driven buoyant flow) are influenced by the gradual increase of wall motion (in terms of Re) due to the added effect of shear flow. These results are discussed systematically in the following sections.

\subsection{Case 1: Both sidewalls cold and moving upwards}

With different speeds of wall motion, the evolution of fluid flow and temperature distribution and energy transport phenomenon for Case 1 (the cold sidewalls move vertically upwards) are shown in figures 3, 4 and 5 for $\operatorname{Re}=10$ and 50, 100 and 200 for $\mathrm{Ri}=0.1-10$, respectively. As both the sidewalls move upward, the shear force on the fluid acts in upward direction, hence wall-adjacent fluid layer is pulled up towards the top corners of the cavity. Additionally, due to the differential heating (as the bottom wall hot and sidewalls cold) of the confined fluid in the cavity, the thermally induced buoyant flow rises upward from the bottom of the cavity. Here, the direction of buoyancy-induced flow as seen from figure 2 , is counteracted by the upward shear flow. At very low $\operatorname{Re}(=10)$ for any value of $\mathrm{Ri} \leq 10$, the flow remains conduction dominated and thermal convection flow is very weak. Hence, the shear flow from the wall motion dictates the flow dynamics in the cavity in the form of clockwise (CW) and counterclockwise (CCW) symmetric circulations about the mid-vertical plane, as shown in the first column of figure 3. Isotherms are distributed from the hot bottom wall showing the height of temperature rise occurs along the midsection of the cavity. Heatline plot for this case shows that the thermal energy transport takes place through the straightforward energy flow corridors from the bottom source to the sidewall sinks. No heat is transferred to the top wall as it is adiabatic.
When $\operatorname{Re}=50$ and strengths of forced convection and free convection are comparable $(\mathrm{Ri}=1)$, the heat transfer is still dominated by conduction. Although, there is no apparent change in fluid flow pattern, the isotherms are more flatter, and depressed downward along the central plane. It happens so as the shear flow passes over the cold sidewalls and deflected downward along the mid-vertical plane. Significant changes are noted in case of energy transport. Energy recirculation cells are formed in the upper region of the cavity as indicated in the second column of the bottom panel. Substantial changes in streamlines, isotherms and heatlines are observed in the last column of figure 3 for $\mathrm{Re}=50$ and $\mathrm{Ri}=10$. Corresponding flow pattern is governed by both the shear flow and buoyancyinduced thermal convection flow, and forms four vortices by splitting the dominating zones of shear flow at $\mathrm{Re}=50$ (indicated by wall adhering vortices) and thermal convection at $\mathrm{Ri}=10$ (resulting $\mathrm{Gr}=2.5 \times 10^{4}$ and indicated by two symmetric middle vortices). Thermal convection causes the rising of hot fluid along the mid-vertical plane of the cavity (similar to earlier shown natural convection case in figure 2a), which is divided at the top wall into leftwards and rightwards forming counterclockwise (CCW) and clockwise (CW) circulation cells, respectively. These circulations are cooled by exchanging heat to the shear-driven vortices at the cold sidewalls. The vortical flow structure in the cavity shows various types of stagnation points as marked by " $S$ " for saddle point and " $E$ " for elliptical point. Bansal and Chatterjee [54] have also reported the existence of saddle and elliptical points in the flow fields of a liddriven cavity. After influenced by upward circulation, isotherms show temperature peaks along the mid-vertical plane. The resulting major energy transport from the bottom wall now follows an upward path and then to either sidewalls. The energy recirculation cells are located at the lower half of the cavity. 


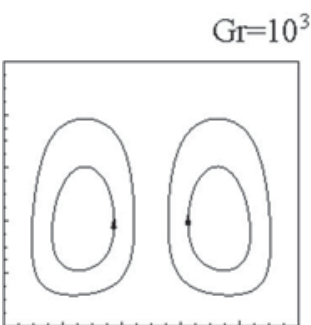

$\mathrm{Gr}=2.5 \times 10^{4}$

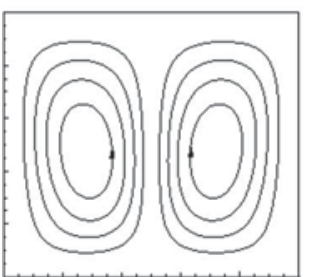

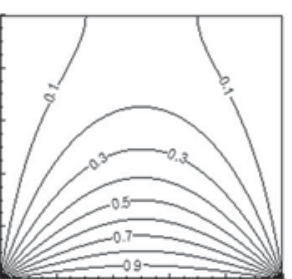

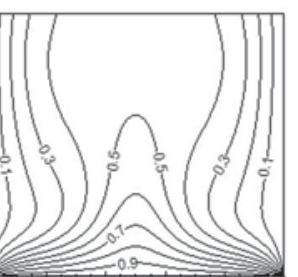

$\mathrm{Gr}=4 \times 10^{3}$

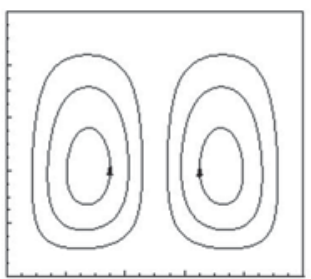

$\mathrm{Gr}=4 \times 10^{5}$
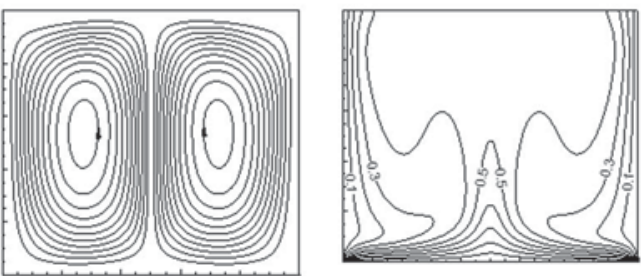

(a) Bottom heated cavity with sidewalls cold and top-wall adiabatic

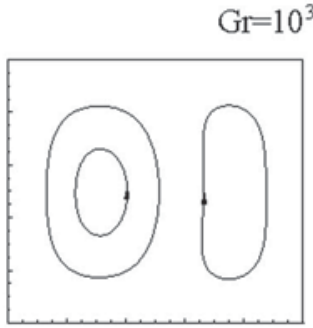

$\mathrm{Gr}=2.5 \times 10^{4}$

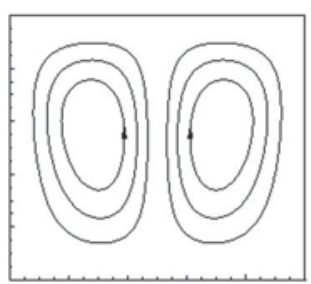

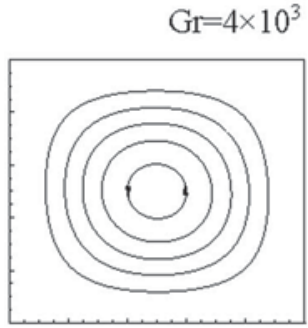

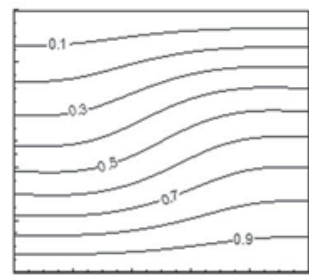

$\mathrm{Gr}=4 \times 10^{5}$
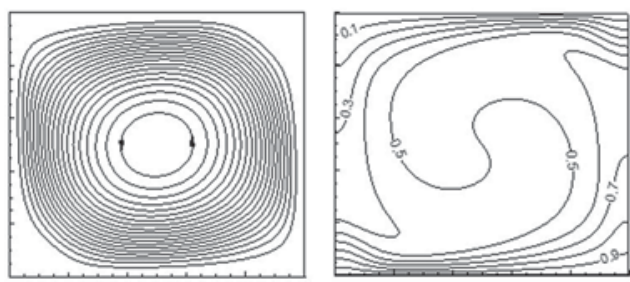

(b) Bottom heated cavity with top-wall cold and sidewalls adiabatic

Figure 2. Flow (streamlines) and thermal (isotherms) patterns in the absence of wall motion.

When $\mathrm{Re}$ is increased to 100 , shown in figure 4 , the streamlines, isotherms and heatlines for $\mathrm{Ri} \leq 1$ show similar trends as found for $\mathrm{Re}=50$ and $\mathrm{Ri}=1$ of figure 3 . However, the enhanced thermal convective circulations and energy recirculation cells are observed at $\mathrm{Re}=100$ and $\mathrm{Ri}=10$ in figure 4, and the size of shear-induced vortices reduces. At further higher speed of wall motion (at $\operatorname{Re}=200$ ), in figure 5, the strength and size of the energy recirculation cells increase appreciably as indicated by the heatlines. At $\mathrm{Ri} \leq 1$, two centrally, downward-moving circulations suppress the formation of thermal convection vortices. The isotherms are pressed downward and localized near the bottom wall. However at $\mathrm{Ri}=10$, the thermal convection flow almost occupies most of the area of the cavity.

\subsection{Case 2: Both sidewalls adiabatic and moving upwards}

The distributions of flow fields due to relocation of thermal boundary condition (sidewalls adiabatic and top-wall cold), keeping upward direction of sidewalls' motion unchanged, are shown for Case 2 in figures 6, 7 and 8 for $\operatorname{Re}=10$ and 50,100 and 200 for $\mathrm{Ri}=0.1-10$, respectively. Here, the differential heating of the confined fluid occurs between the 

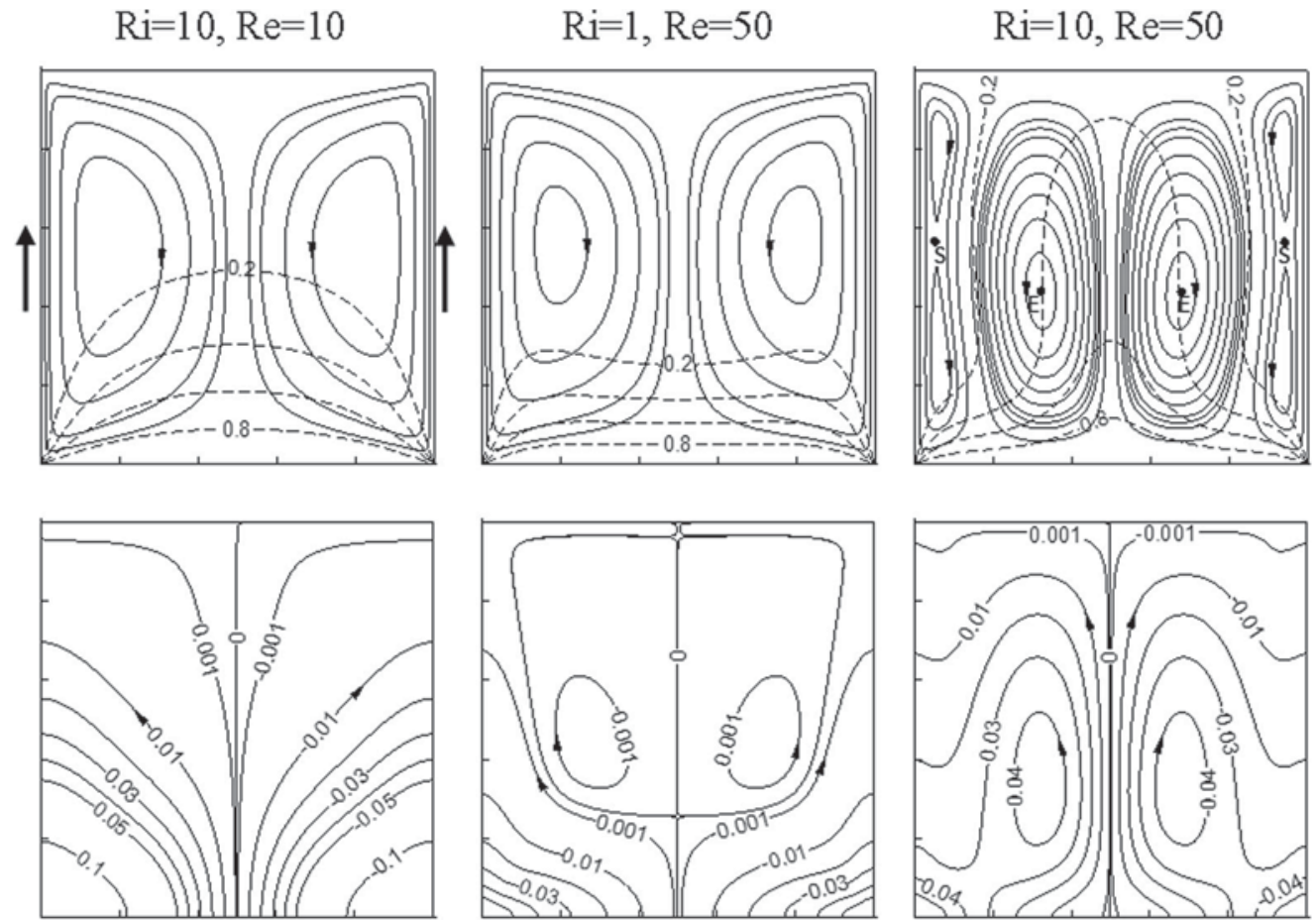

Figure 3. Streamlines and isotherms (top panel), and heatlines (bottom panel) of Case 1 .
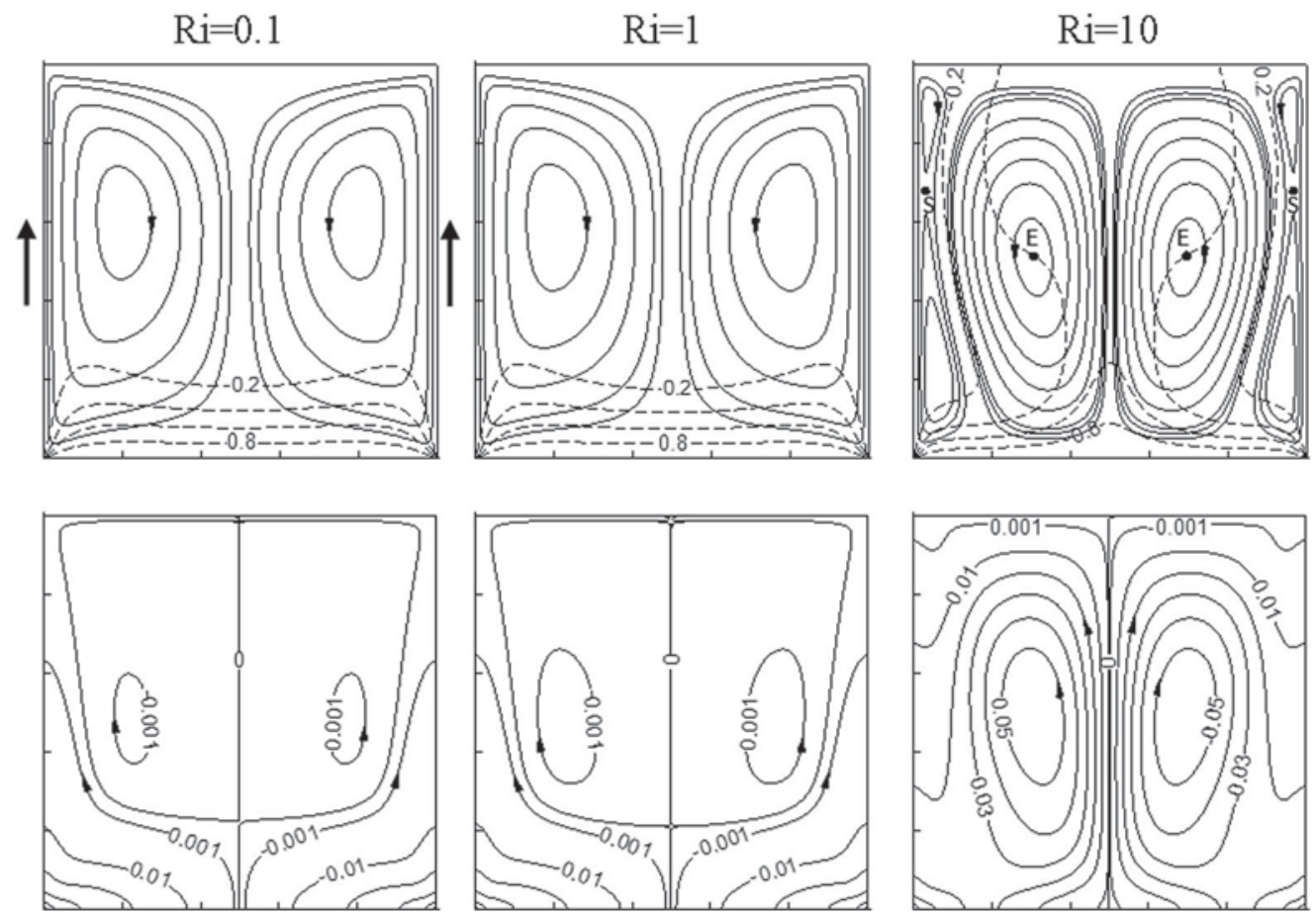

Figure 4. Streamlines and isotherms (top), and heatlines (bottom) of Case 1 at $\operatorname{Re}=100$.

hot bottom wall and the cold top wall, as the sidewalls are adiabatic. For this thermal boundary configuration in the absence of the wall motion, stratified form of temperature distribution is noted along with $\mathrm{CCW}$ and $\mathrm{CW}$ circulations (from left to right region of the cavity) at lower $\mathrm{Gr}$ in figure $2 \mathrm{~b}$. When the upward translation of the sidewalls is 

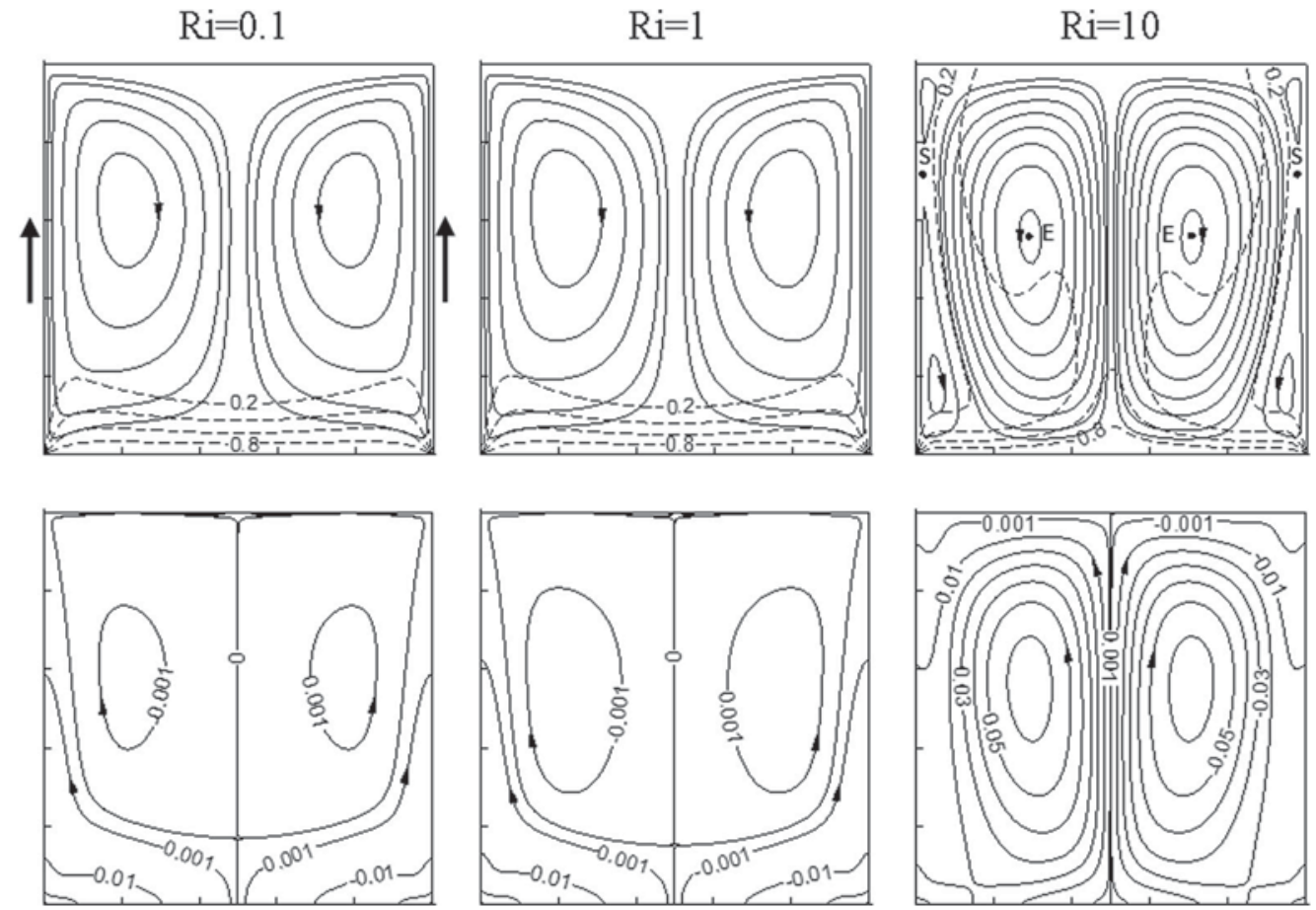

Figure 5. Streamlines and isotherms (top), and heatlines (bottom) of Case 1 at $\operatorname{Re}=200$.
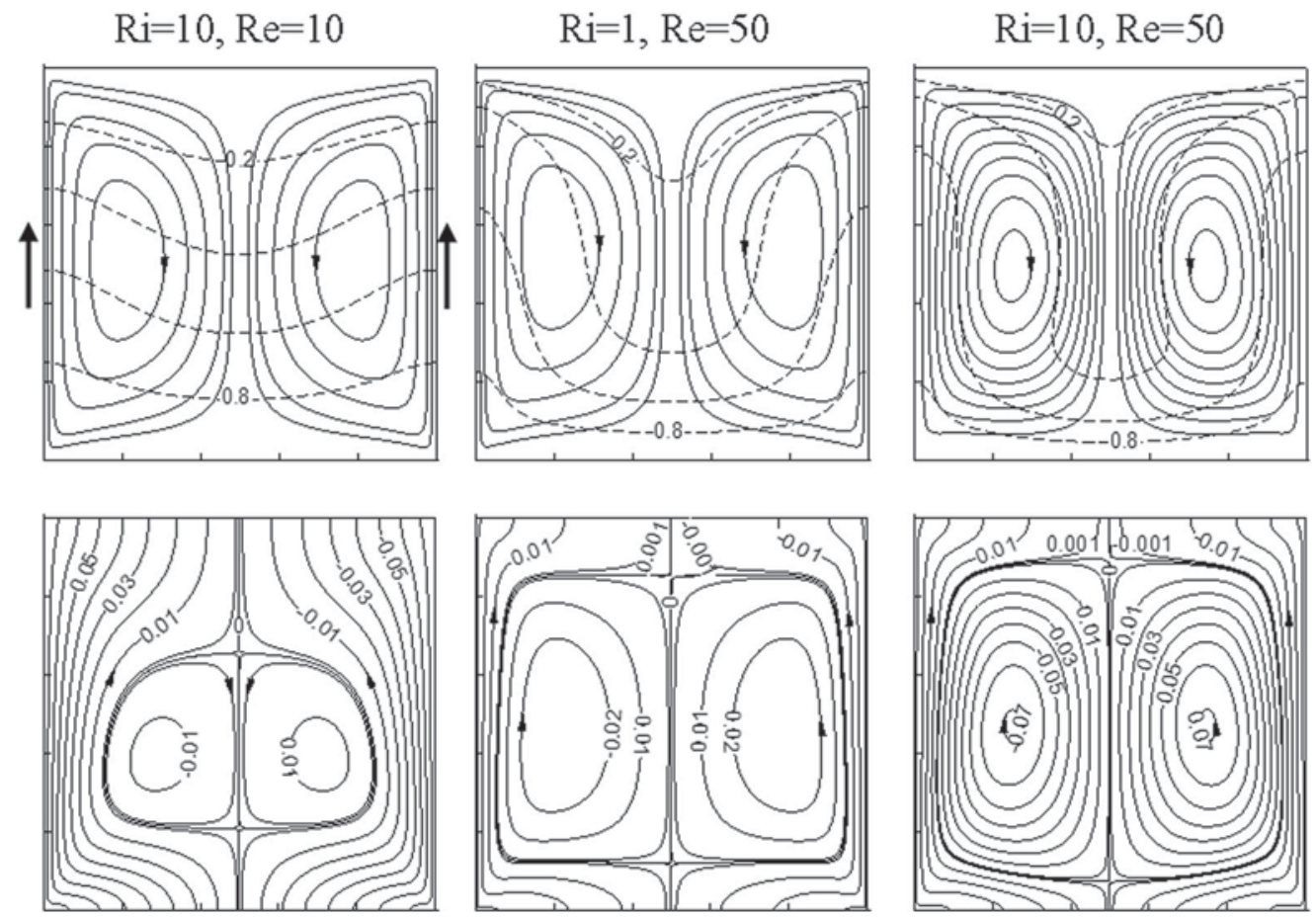

Figure 6. Streamlines and isotherms (top panel), and heatlines (bottom panel) of Case 2.

applied, the heated fluid adjacent to the bottom wall is driven towards the bottom of either sidewalls and flows upwards along the sidewalls as shown in figure 6. The hotter fluid releases heat at the cold top wall. The cooling persists along the mid-vertical plane downward from the top wall. The ends of isotherm contours are shifted upwards 

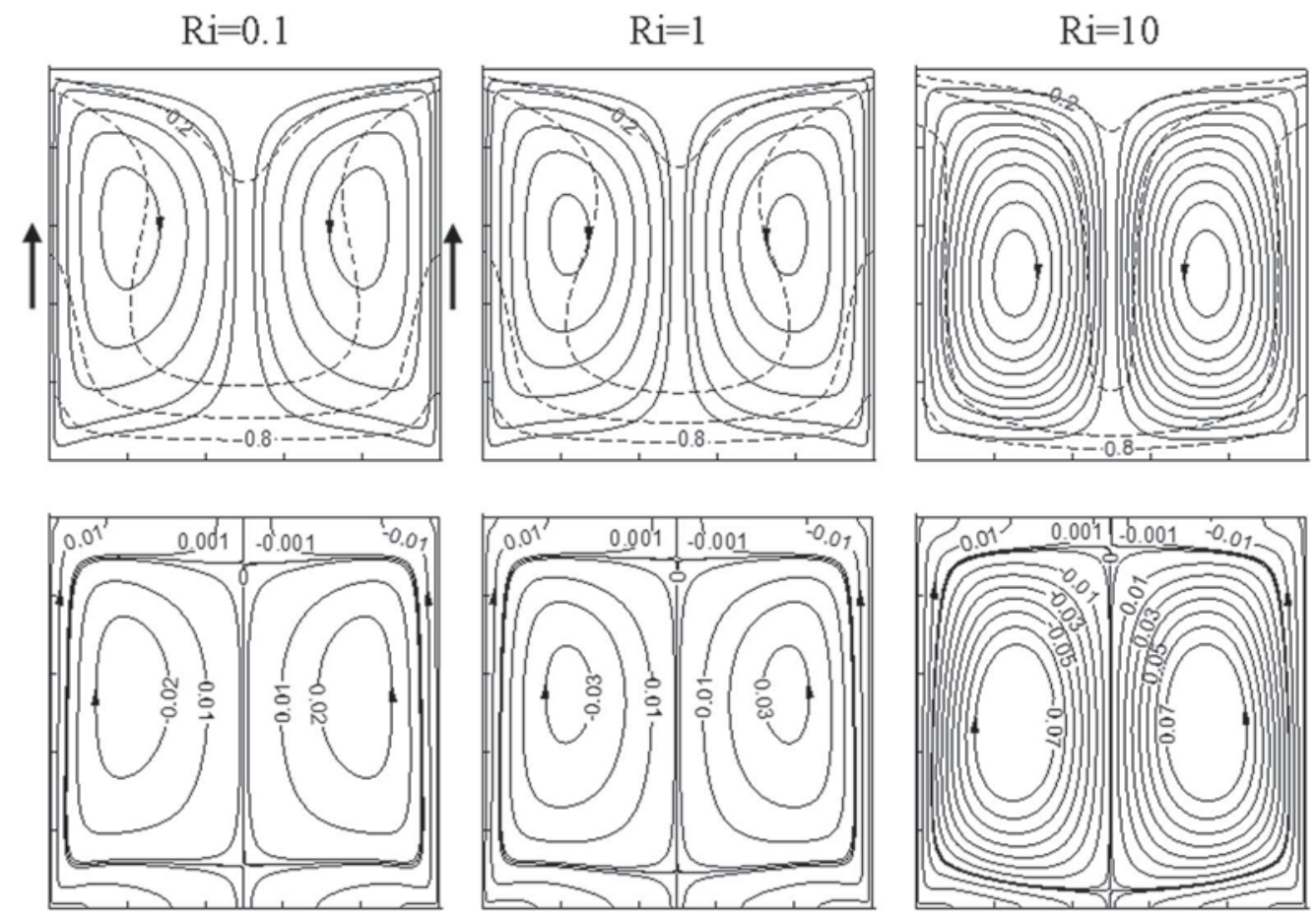

Figure 7. Streamlines and isotherms (top), and heatlines (bottom) of Case 2 at $\operatorname{Re}=100$.
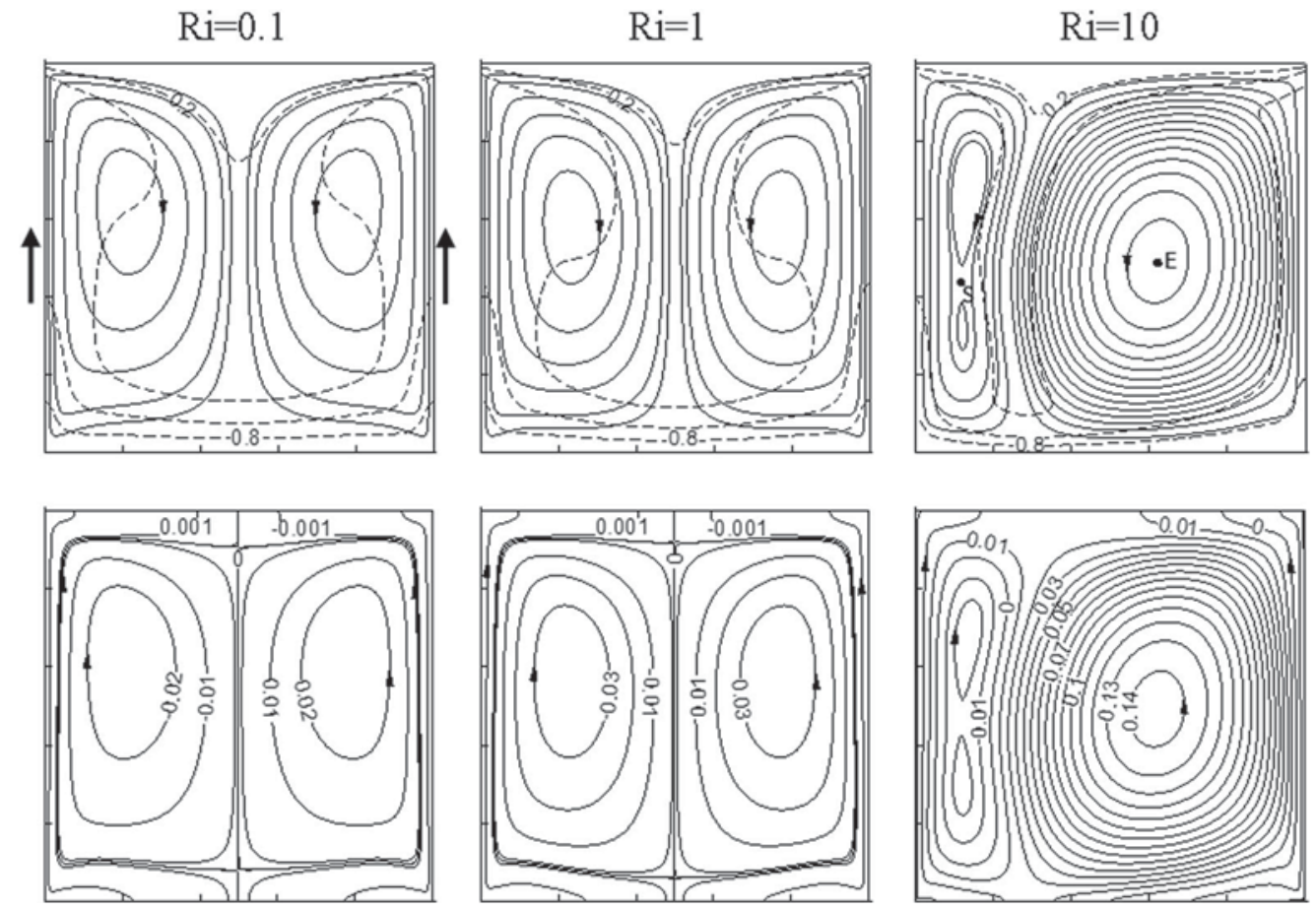

Figure 8. Streamlines and isotherms (top), and heatlines (bottom) of Case 2 at $\operatorname{Re}=200$.

according to the speed of sidewalls and their middle portion is depressed downwards. The confined fluid rotates in the form of $\mathrm{CW}$ and $\mathrm{CCW}$ circulations (from left to right) for all $\operatorname{Re} \leq 50$ and $\mathrm{Ri} \leq 10$. The heatline plots show that the energy recirculation cells are located at the central region of the cavity and occupied most of the portion of the cavity at 
$\mathrm{Re}=50$ and $\mathrm{Ri}=10$. The energy transport between the hot and cold walls takes place over the energy recirculation cells symmetrically. The strength and size of the energy recirculation cells are found to increase markedly. Only elliptical stagnation points are located at the centre flow vortices.

In figure 7 , at $\mathrm{Re}=100$ for different $\mathrm{Ri}$ values, the patterns of streamlines, isotherms and heatlines are found to be similar. Stronger circulation and energy recirculation are observed at $\mathrm{Ri}=10$ due to enhanced thermal convective flow. The symmetrical transports of mass and energy (about the mid-vertical plane) are found to be lost at higher Re and $\mathrm{Ri}$ as presented in figure 8, particularly at $\mathrm{Re}=200$ and $\mathrm{Ri}=10$. The right $\mathrm{CCW}$ vortex is much larger compared with the left $\mathrm{CW}$ vortex. This is a peculiar flow feature and it evolves due to combined effect of stronger thermal convection and shear flow. In the absence of the thermal convection, the circulations due to wall-induced shear flow are symmetric. On the other hand, in the absence of the shear flow and at higher $\mathrm{Gr}$, a single large $\mathrm{CCW}$ vortex is observed as shown in figure $2 \mathrm{~b}$. The combination of these two effects (two CW and CCW symmetrical shear vortices, and single $\mathrm{CCW}$ thermal convection vortex) results in the asymmetrical vortical patterns observed in figure 8. At lower $\mathrm{Ri}(\leq 1)$ with $\operatorname{Re}=200$, this asymmetrical trend is not pronounced as $\mathrm{Gr}<10^{5}$. The heatline plots of energy transport follow a similar pattern of streamline plots, as the heatlines depend on both convective energy flux and conductive energy flux.

\subsection{Case 3: Both sidewalls cold and moving downwards}

In contrast with the upward translation of sidewalls as discussed for Case 1, here, downwards motion of cold sidewalls favours the formation of $\mathrm{CCW}$ and $\mathrm{CW}$ circulations in the cavity as shown in figures 9, 10 and 11 for $\mathrm{Re}=10$ and 50, 100 and 200 for $\mathrm{Ri}=0.1-10$, respectively. In this case, the wall-driven shear flows always assist the thermal circulations. Irrespective of $\mathrm{Re}-\mathrm{Ri}$ values, the streamlines, isotherms and heatlines are symmetrically distributed about the mid-vertical plane.

In figure 9, the heat from bottom wall is being carried upwards along the middle of the cavity. At $\operatorname{Re}=10$ and $\mathrm{Ri}=10$, the heatlines go upwards, then they are turned laterally either sidewalls. The closed isotherms get opened and becomes vertical as Re increases to 50. The concentrated streamlines at $\mathrm{Re}=50$ and $\mathrm{Ri}=10$, indicate the development of higher circulation velocity in the cavity. Corresponding heatlines show the formation of energy recirculation cells. The intensity of energy flux at the central region is stronger due to higher convective flow (from both shear flow and thermal convection). The heatline plots show the general trend of energy distribution from the bottom source to the sidewall sinks; for example the heat from the middle portion of the bottom wall is transported to the upper portion.

As wall speed (or Re) increases, in figures 10 and 11, the vertical isotherm contours are more deflected from the central axis towards sidewalls. Along the sidewalls, the formation of thinner thermal boundary layer is noticeable at $\mathrm{Ri}=10$. The energy transport from the ends of the bottom wall to the sidewalls takes place following a longer path in the form of a loop, which is clearly marked in figure 10 . The corner connectivity of heatlines is not visible in this case. Here, the circulations (both $\mathrm{CCW}$ and $\mathrm{CW}$ ) are much stronger as $\mathrm{Gr}$ is high $\left(4 \times 10^{5}\right)$.

\subsection{Case 4: Both sidewalls adiabatic and moving downwards}

The thermal conditions of sidewalls and top wall of Case 3 are interchanged without affecting the direction of the wall motion. Corresponding evolutions of mass and energy transports are shown figures 12, 13 and 14 for $\mathrm{Re}=10$ and 50, 100 and 200 for $\mathrm{Ri}=0.1-10$, respectively. For this case also, the wall-driven shear flows assist the thermal circulations. The formation of symmetrical patterns about vertical central axis is found for all streamlines, isotherms and heatlines irrespective of Re-Ri values.

The heatlines in figure 12 show the direct, upwards transport of thermal energy from the hot bottom wall to the cold top wall. The maneuvering of heat transport shows the connectivity of similar parts of the source and the sink (the source's ends connect to the sink's ends, the source's middle part connect to the sink's middle part). The isotherms become convex for $\mathrm{Re}=10$ and $\mathrm{Ri}=10$ as the sidewalls translate downwards. As Re increases to 50, the isotherm contour 0.8 near the bottom wall forms a prominent peak at its centre. At higher Re of 100 or 200, when $\mathrm{Ri} \leq 1$, marginal changes in the contour distributions of flow fields are observed in figures 13 and 14, respectively as correspond thermally induced convection is not so high. But at $\mathrm{Ri}=10$, significant changes are noticed: stronger mass circulations and energy recirculations exist. Large heatline cells at each half of the cavity are formed due to the enhanced convection. The corresponding zone of more uniform temperature between 0.6 and 0.4 is found to be very large.

\subsection{Comparative assessment on mid-plane velocity and temperature}

The effects of Richardson number, direction and magnitude of wall motion and position of cold wall(s) on the flow fields as well as on thermal fields are summarized using the non-dimensional quantities about the mid-horizontal plane in figure 15 for $\operatorname{Re}=50$ and 200. The vertical component 

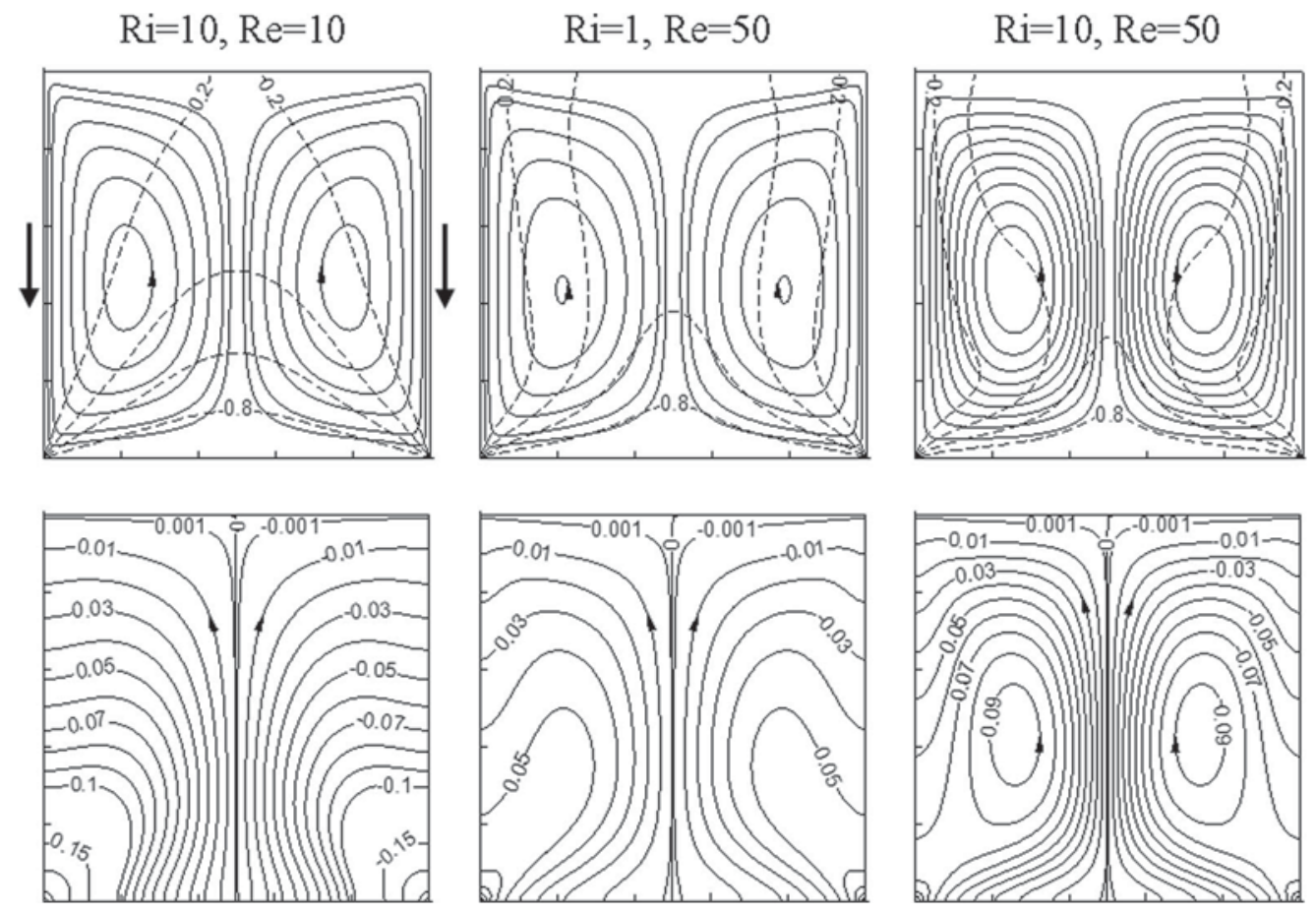

Figure 9. Streamlines and isotherms (top panel), and heatlines (bottom panel) of Case 3.
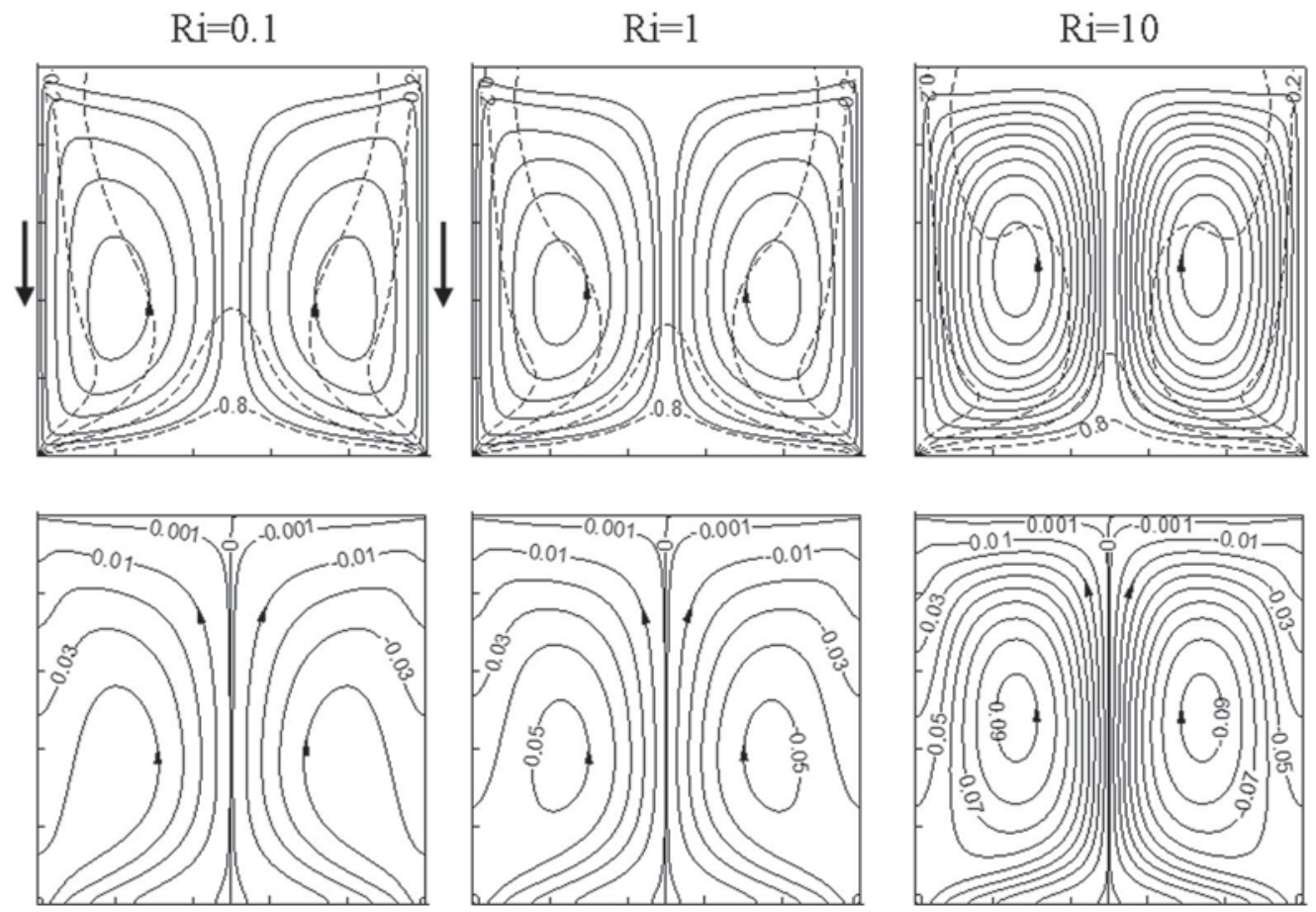

Figure 10. Streamlines and isotherms (top), and heatlines (bottom) of Case 3 at $\operatorname{Re}=100$.

of velocity $(V)$ along the mid-horizontal plane indicates that at sidewalls (as they move up or down), $V$ becomes +1.0 for cases 1 and 2 and -1.0 for cases 3 and 4, irrespective of Re and Ri. However, across the cavity, $V$ changes (i.e., reduces or increases) depending upon $\mathrm{Re}-\mathrm{Ri}$ values and direction of wall motion. $V$ curves at $\mathrm{Ri} \leq 1$ are found almost coinciding due to weaker thermal convection irrespective of flow in cases 1-4. 

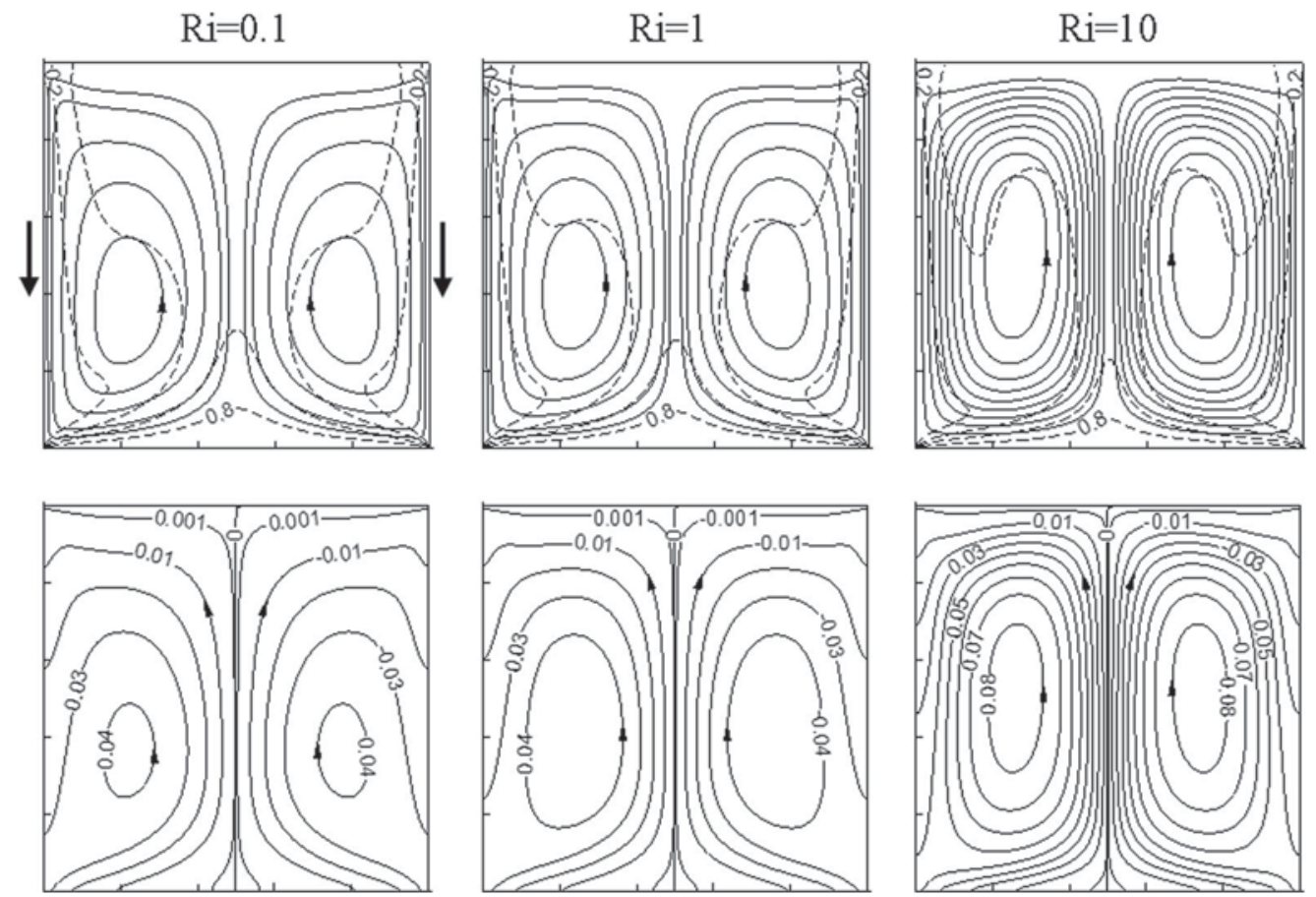

Figure 11. Streamlines and isotherms (top), and heatlines (bottom) of Case 3 at $\operatorname{Re}=200$.
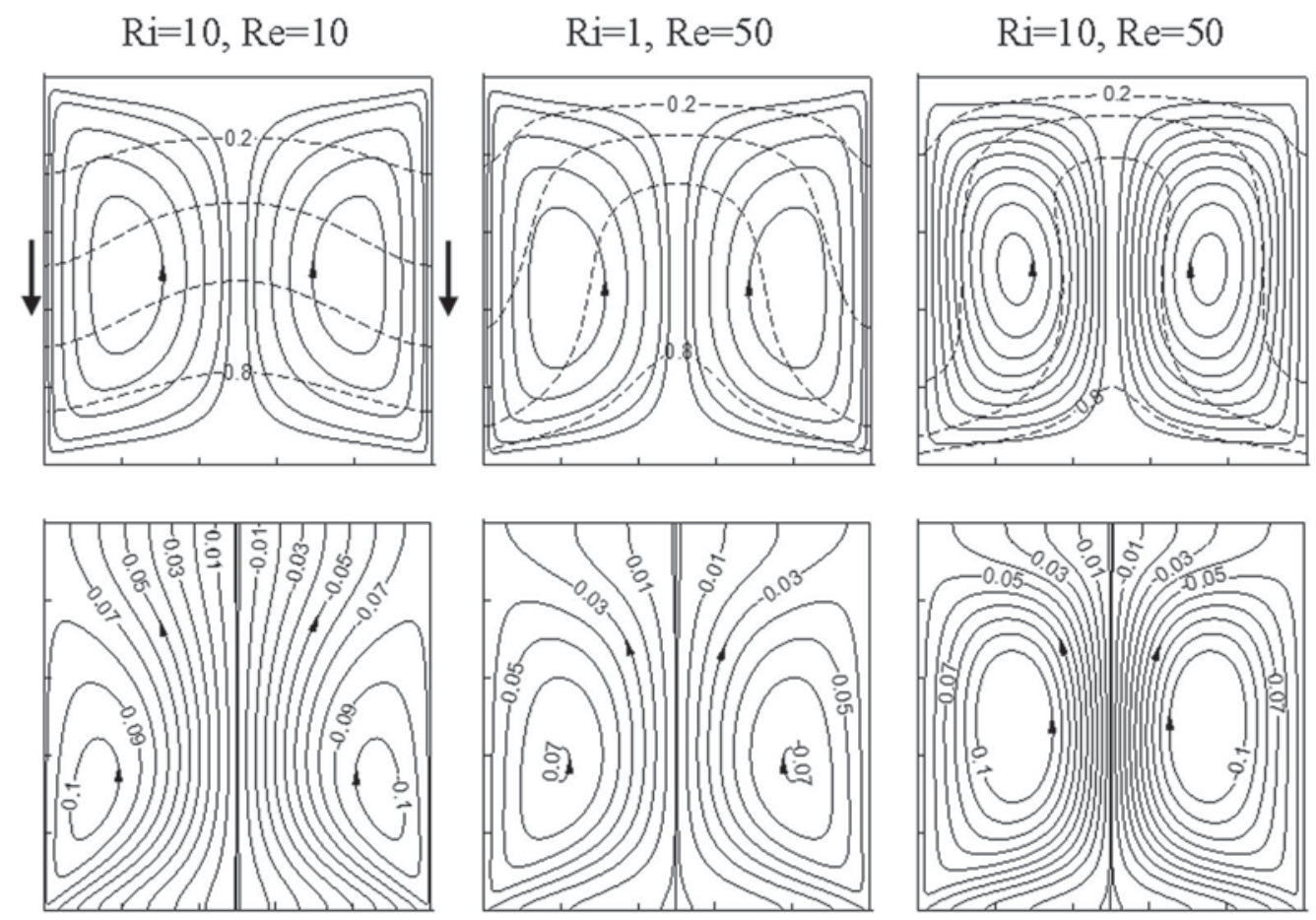

Figure 12. Streamlines and isotherms (top panel), and heatlines (bottom panel) of Case 4.

For Case 1, with cold sidewalls and adiabatic top wall at higher $\mathrm{Ri}(\geq 10), V$ profiles look sinusoidal, with decreasing trend near sidewalls. It refers to flow patterns at $\mathrm{Ri}=10$ as shown in figures 3, 4 and 5 with four flow vortices. Here, it is interesting to note that the directions of fluid circulations change as $\mathrm{Ri}$ increases. 

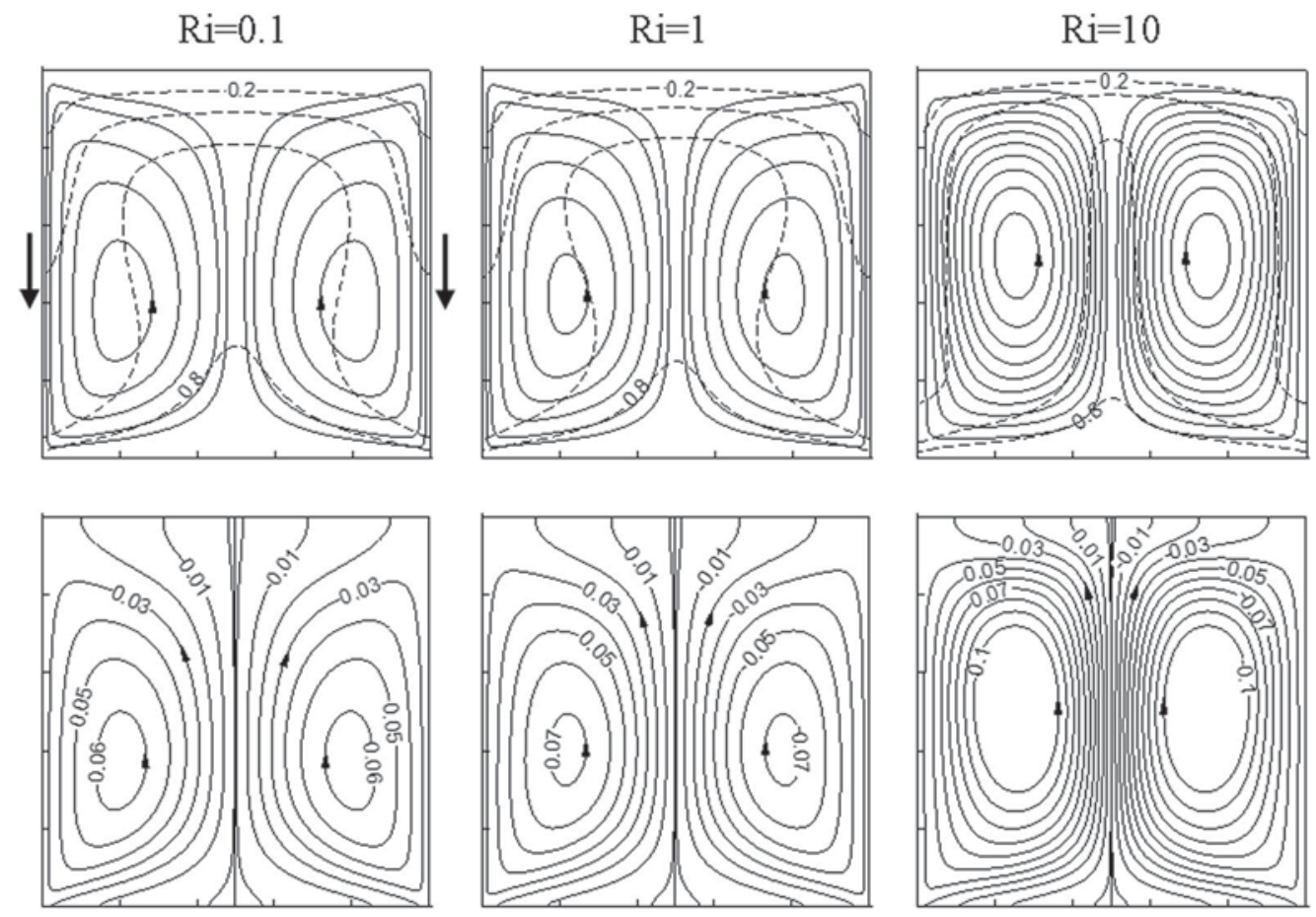

Figure 13. Streamlines and isotherms (top), and heatlines (bottom) of Case 4 at $\operatorname{Re}=100$.
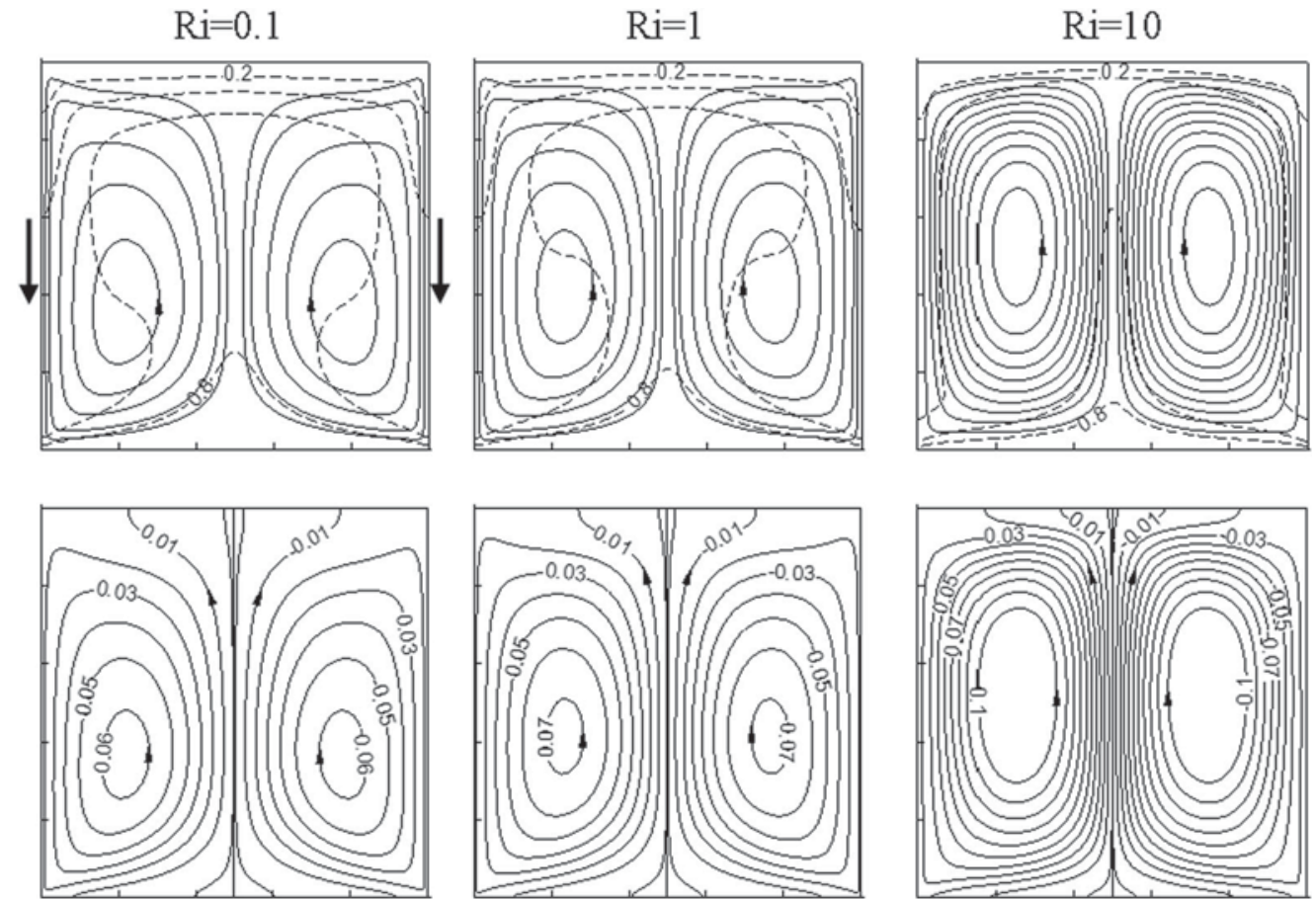

Figure 14. Streamlines and isotherms (top), and heatlines (bottom) of Case 4 at $\mathrm{Re}=200$.

Downwards flow velocity at centre at observed for lower $\mathrm{Ri}$ is gradually overcome as thermal convection becomes stronger with increasing $\mathrm{Ri}$. At $\mathrm{Ri}=100$,
$V$ markedly increases along the mid-vertical plane. However, the flow patterns are found to be symmetric for all Re and Ri. 
$\boldsymbol{V}$

$\theta$

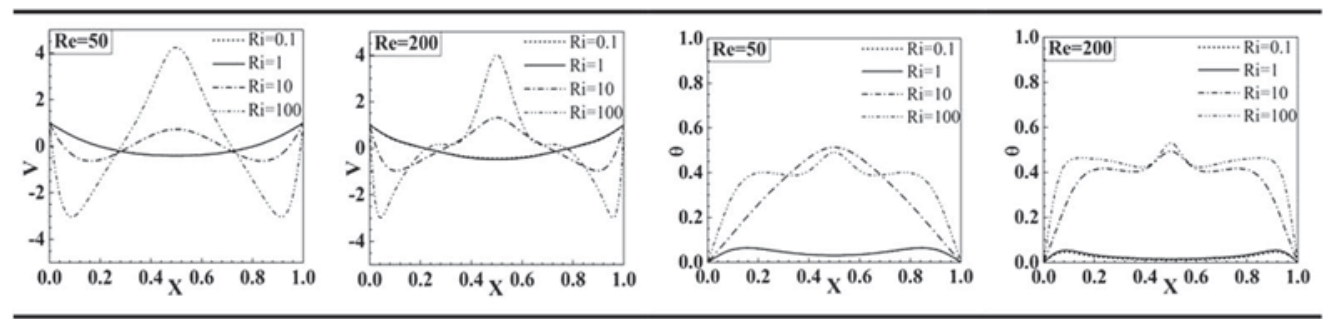

Case 1

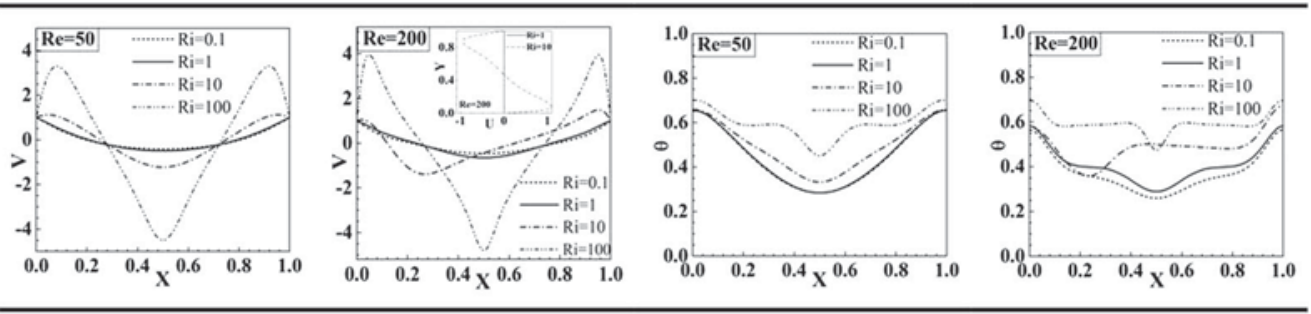

Case 2

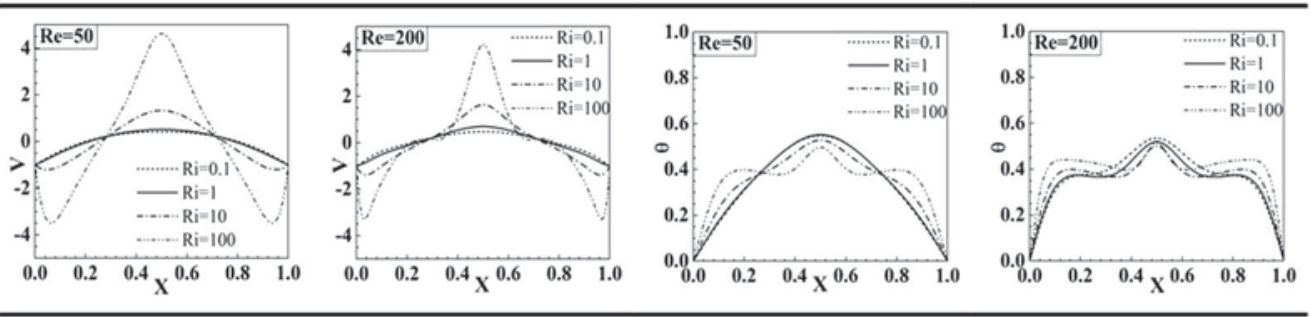

Case 3
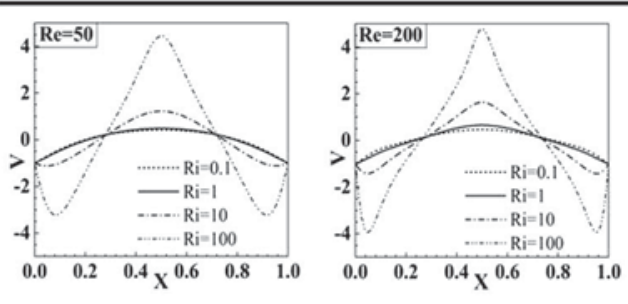

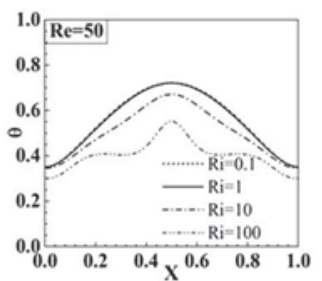

Case 4

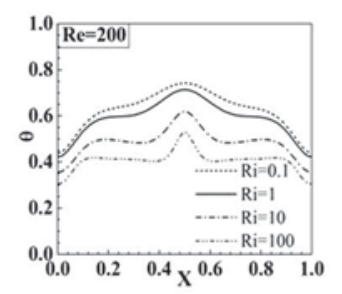

Figure 15. Variations of mid-plane velocity and temperature for different flow cases.

For Case 2, with cold top wall and adiabatic sidewalls at higher Ri ( $\geq 10$ ) although $V$ profiles look sinusoidal, they show opposite trends compared with Case 1. Here, direction of fluid rotation remains unchanged with $\mathrm{Ri}$. As $\mathrm{Ri}$ increases, $V$ significantly increases near the sidewalls and markedly decreases along the mid-vertical plane. It refers to streamline plots shown in figures $6,7,8$ and 9 with two vortices. The flow pattern is found to be asymmetric only at $\mathrm{Re}=200$ and $\mathrm{Ri}=10$. Two zero-values of $V$ refers the existence of two flow vortices; left $\mathrm{CW}$ vortex which is small compared to large $\mathrm{CCW}$ vortex located at right; corresponding flow visualization is shown in figure 8. At higher Ri value $(=100)$, the feature of flow asymmetry diminishes. It can be better understood from horizontal velocity component $(U)$. $U$ curve (as shown in the inset of
Case 2) along the mid-vertical plane is found asymmetric only at $\mathrm{Ri}=10$ and $\mathrm{Re}=200$. Except this, $U$ plots are straight vertical line with zero value, which indicates symmetric nature about the mid-vertical plane. Both the negative and positive peak velocities are substantially higher at $\mathrm{Ri}=100$, irrespective of $\mathrm{Re}=50$ or $\mathrm{Re}=200$.

For cases 3 and 4, with downwards wall motion, the velocity profiles are found to be similar. $V$ at centre bearing positive values for all $\mathrm{Re}$ and Ri. Velocity profiles are symmetric and peak velocity markedly increases as $\mathrm{Ri}$ increases. Incidentally $V$ profiles at higher Ri are similar to those of Case 1 also.

The non-dimensional temperature $(\theta)$ plots for Case 1 show very small $\theta$ values for Ri $\leq 1$ irrespective of Re. At higher $\mathrm{Ri}$ from the cold walls of zero temperature, 
significant rise of $\theta$ is noted for the middle extent of the curve. This is due to mid-center upward flow velocity. However for Case 2, when the sidewalls are set adiabatic, appreciable rise in temperature is found across the cavity. consistent with $V$ profile, $\theta$ profile is asymmetric at $\mathrm{Re}=200$ and $\mathrm{Ri}=10$. The temperature decreases from both side walls and reaches the minimum at $\mathrm{X}=0.5$ and this pattern is similar for all Ri. Temperature variation for Case 3 is found to be opposite to that of Case 1. For Case 4, the $\theta$ profile indicates the presence of higher temperature near the sidewalls, which further increases till $X=0.5$ for any $\mathrm{Re}-\mathrm{Ri}$ value. Another important point is that the position of cold wall at the cavity top increases the temperature of the fluid near adiabatic sidewalls (Cases 2 and 4); as the heat transfer area through the cold wall is half of that of Cases 1 and 3, it causes less heat transport. The results corresponding to higher wall velocity at $\operatorname{Re}=200$ show more sharp changes in $\mathrm{V}$ plots as could be observed in figure 15.

Overall heat transfer characteristics for the present investigation are plotted in figure 16. Average Nusselt number $\left(\mathrm{Nu}_{\mathrm{avg}}\right)$ is computed for the hot bottom wall. The trends of $\mathrm{Nu}_{\text {avg }}$ curves against Richardson number, $\mathrm{Ri}$, are found to be increasing gradually irrespective of specific case and Re value. The rate of increment is higher at higher $\mathrm{Ri}$. $\mathrm{Nu}_{\mathrm{avg}}$ also increases with $\mathrm{Re}$ for all the cases under consideration. With regard to specific cases, $\mathrm{Nu}_{\mathrm{avg}}$ for Case 3 are higher compared with all other cases and with $\mathrm{Re}=200$, significant increase in $\mathrm{Nu}_{\mathrm{avg}}$ is observed. The heat transfers for Cases 2 and 4 are comparable.

In general, the heat transfer is found to increase with $\mathrm{Ri}$ due to stronger buoyancy force with increasing Ri (particularly after $\mathrm{Ri}>1$ ). At $\mathrm{Ri}<1$, the heat transfer is mainly governed by the wall motion or forced convection. There are no significant changes in overall heat transfer characteristics of Cases 2 and 4. For both these cases, the top wall acts as the sink for heat rejection. Due to the reduced cooling length (compared with cold sidewalls cases), the heat transfer is lower than in the the sidewall-cooling cases (Cases 1 and 3 ). Case 3 shows maximum heat transfer.
With respect to it (Case 3), Case 1, next higher heat transferring case, transports 3-17\% (depending upon Re (50-200) and $\mathrm{Ri}(0.1-100)$ values) lesser amount of thermal energy from the bottom wall. Both Cases 1 and 3 have identical cooling surfaces (as cooled by both sidewalls). For Cases 2 and 4, the reduction in heat transfer with respect to Case 3 is around $50 \%(\operatorname{Re}=200)$ to $76 \%(\operatorname{Re}=50)$. According to the thermal boundary configuration, with 50\% difference in cooling surface area, the heat transfer for Case 2 is $46-76 \%$ less compared to Case 1, and it for Case 4 is 50-76\% less compared with Case 3, over the ranges of $\mathrm{Re}=50-200$ and $\mathrm{Ri}=0.1-100$. These facts establish the strong positional impact of the cold wall and direction of the moving walls on the heat transfer.

\subsection{Effect on thermal mixing}

The order of thermal mixing for the present problem is analysed using both velocity weightage temperature or cupmixing temperature $\left(\theta_{\text {cup }}\right)$ and volume weightage temperature or bulk-averaged temperature $\left(\theta_{\text {avg }}\right)$. Their expressions for two-dimensional flow are expressed in dimensionless form as

$$
\begin{gathered}
\theta_{\text {cup }}=\frac{\iint \widehat{V}(X, Y) \theta(X, Y) d X d Y}{\iint \widehat{V}(X, Y) d X d Y}, \text { where } \widehat{V}=\sqrt{U^{2}+V^{2}} \\
\theta_{\text {avg }}=\frac{\iint \theta(X, Y) d X d Y}{\iint d X d Y}
\end{gathered}
$$

Here, the double integrations are carried out over the entire flow domain. Similar expressions are also mentioned in [56].

The results of cup-mixing temperature $\left(\theta_{\text {cup }}\right)$ and bulkaveraged temperature $\left(\theta_{\text {avg }}\right)$ are shown in figure 17 at a fixed Ri value for different cases against varying Re values. They are indicated by different curves with wide differences (approximately 0.1-0.6) particularly at lower Ri. At
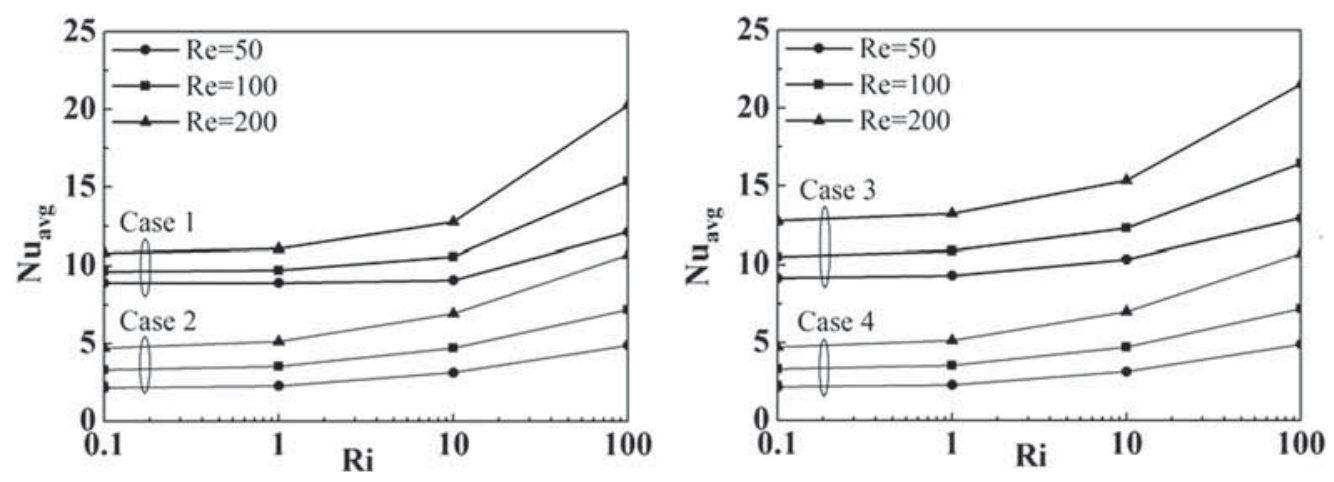

Figure 16. Average Nusselt number $\left(\mathrm{Nu}_{\mathrm{avg}}\right)$ for different flow cases. 

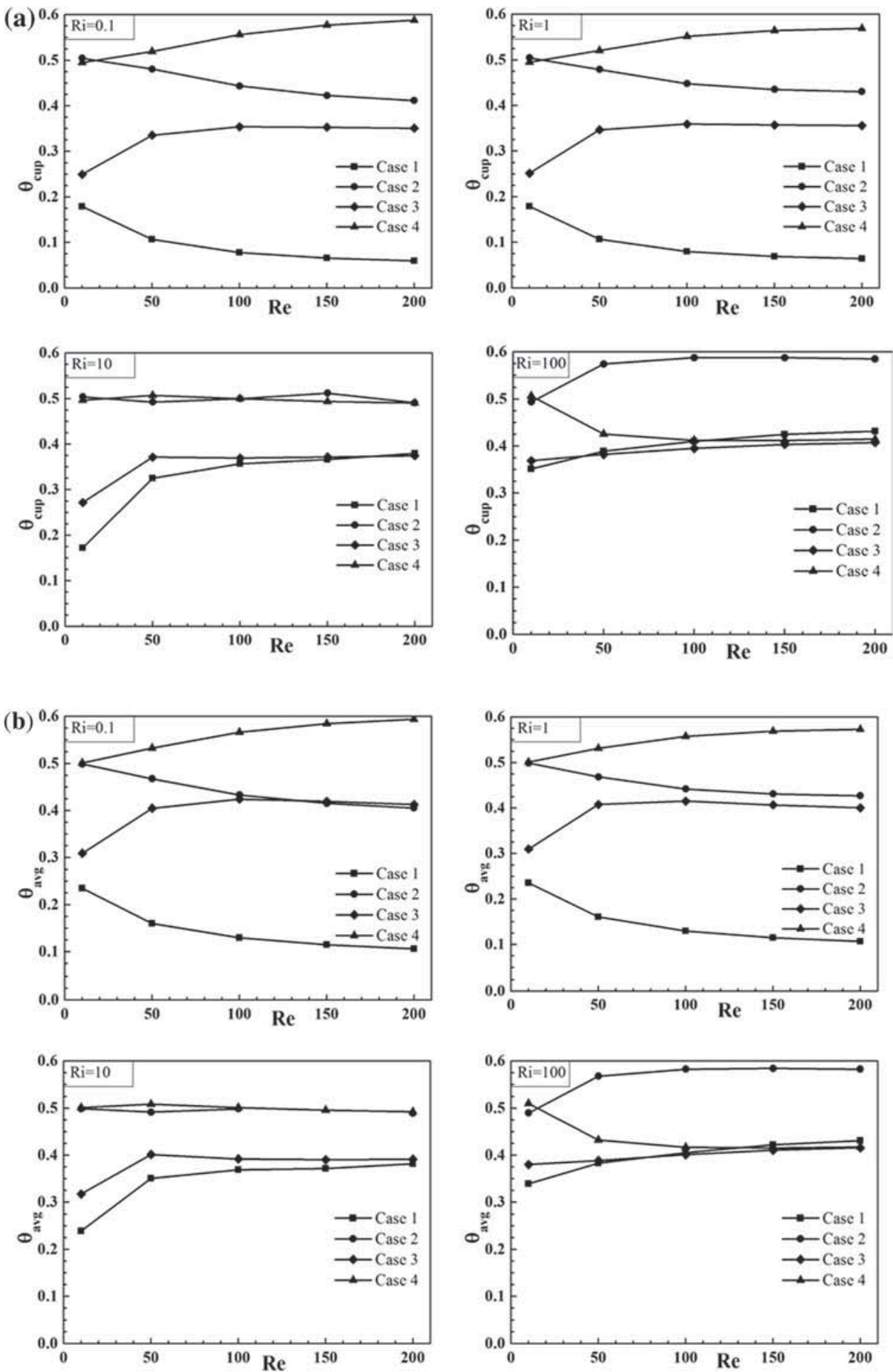

Figure 17. Order of thermal mixing for different flow cases: (a) $\theta_{\text {cup }}$ and (b) $\theta_{\text {avg }}$.

lower $\mathrm{Ri}(\leq 1)$ with the increase of Re, Cases 1 and 2 show decreasing trends when the sidewalls are translating upwards, whereas Cases 3 and 4 show increasing trends when the sidewalls are translating downwards; $\theta_{\text {cup }}$ and $\theta_{\text {avg }}$ are the lowest for, respectively, Case 1 and the highest for Case 4.
At higher Ri (=10-100), the nature of curves, except for $\operatorname{Re}=10$, is almost flat. At $\mathrm{Ri}=10$, curves of Cases 1 and 3 , and Cases 2 and 4 are almost coinciding, showing orders of $\theta_{\text {cup }}$ around 0.35 and 0.5 , respectively. At Ri $=100, \theta_{\text {cup }}$ becomes maximum $(\sim 0.6)$ with Case 2 , rest of the three 
cases are coinciding $\left(\theta_{\text {cup }} \sim 0.4\right)$ after $\operatorname{Re}=50$. Similar observations at higher $\mathrm{Ri}$ are also noted for $\theta_{\text {avg }}$.

Above results indicate that $\theta_{\text {cup }}$ of Case 2 with sidewalls adiabatic and moving upwards is most sensitive to $\mathrm{Ri}$ value and increases consistently with Ri. In contrast, $\theta_{\text {cup }}$ of Case 4 with sidewalls adiabatic and moving downwards, which carries the highest value at lower Ri, gradually decreases with Ri value. In general, both $\theta_{\text {cup }}$ and $\theta_{\text {avg }}$ for Cases 4 and 2 are found to be consistently higher compared with Cases 3 and 1, which can be realized from the temperature profile plot shown in figure 15 .

\section{Conclusions}

The transport phenomena in a side-wall moving, bottomheated cavity are explored considering both upwards and downwards motion of sidewalls and alternation of cooling between sidewalls and top wall (four different cases). The study demonstrates the transport of thermal energy from the heated bottom of the cavity utilizing Bejan's heatlines and the pattern of induced-fluid flow using streamlines, under different magnitudes of flow-controlling parameters ( $\mathrm{Ri}$ and $\mathrm{Re}$ ). Thermal performance under different cases is estimated to understand their relative efficacies. Major observations are summarized below.

- Both the direction of sidewall translation and thermal conditions of top and side walls play major roles in transporting thermal energy. Adiabatic top wall and cold sidewalls show higher rate of heat transfer irrespective of direction of wall motion. This may be due to the greater cooling surface from both the sidewalls. Direction of wall motion controls the rotation of vortices and causes energy flow from the source to be inwards through the centre or to be outwards along the sidewalls.

- Under forced and mixed convection regimes $(\mathrm{Ri} \leq 1)$, the heat transfer is found to be almost constant. It increases at higher Ri $(>1)$, when natural convection plays a dominant role. Average Nusselt number markedly increases at $\mathrm{Ri}=100$.

- For each case of the thermal conditions and wall motions, the heat transfer at a fixed $\mathrm{Ri}$ is found to increase with higher wall speed (or higher Re), which is due to stronger buoyancy force (or Gr) with increasing Re.

- Heat transport from bottom wall is found to be more when cold sidewalls move downwards. For top-cooling arrangement, the direction of sidewall motion has insignificant impact on the heat transfer.

- Both $\theta_{\text {cup }}$ and $\theta_{\text {avg }}$ are comparatively much higher for Cases 2 and 4. For Cases 1 and 2, the cup-mixing temperature $\left(\theta_{\text {cup }}\right)$ decreases with $\mathrm{Re}$ for $\mathrm{Ri} \leq 1$ and it increases for $\mathrm{Ri}>1$, whereas it is almost invariable in Case 3. For Case 4, cup-mixing temperature $\left(\theta_{\text {cup }}\right)$ increases with $\mathrm{Re}$ for $\mathrm{Ri} \leq 1$ and then decreases with $\mathrm{Re}$ for $\mathrm{Ri}>1$. The order of thermal mixing within the cavity increases with increasing Ri.

The outcome of the present work can be useful for the thermal design of a similar system. Furthermore, this knowledge of insight details could be treated as the base case for extending study involving multi-physical flow situations like porous media, nanofluids, ferrofluids, debris cooling, heat generation, magnetohydrodynamics, etc.

\section{Nomenclature}

$\begin{array}{ll}g & \text { acceleration due to gravity }\left(\mathrm{m} \mathrm{s}^{-2}\right) \\ \mathrm{Gr} & \text { Grashof number } \\ L & \text { length scale }(\mathrm{m}) \\ \mathrm{Nu} & \text { Nusselt number } \\ p & \text { pressure }(\mathrm{Pa}) \\ P & \text { dimensionless pressure } \\ \mathrm{Pr} & \text { Prandtl number } \\ \mathrm{Re} & \text { Reynolds number } \\ \mathrm{Ri} & \text { Richardson number } \\ T & \text { temperature }(\mathrm{K}) \\ u, v & \text { velocity components }\left(\mathrm{m} \mathrm{s}^{-1}\right) \\ v_{w} & \text { velocity of the moving wall }\left(\mathrm{m} \mathrm{s}^{-1}\right) \\ U, V & \text { dimensionless velocity components } \\ x, y & \text { Cartesian coordinates (m) } \\ X, Y & \text { dimensionless coordinates }\end{array}$

\section{Subscripts}

a ambient

avg average

$h$ hot wall

$c \quad$ cold wall

cup cup-mixing temperature

\section{References}

[1] Bettaibi S, Sediki E, Kuznik F and Succi S 2015 Lattice Boltzmann simulation of mixed convection heat transfer in a 
driven cavity with non-uniform heating of the bottom wall. Commun. Theor. Phys. 63(1): 91-100

[2] Bond D, Goldsworthy M J and Wheatley V 2015 Numerical investigation of the heat and mass transfer analogy in rarefied gas flows. Int. J. Heat Mass Transf. 85: 971-986

[3] Cheng T S and Liu W -H 2014 Effects of cavity inclination on mixed convection heat transfer in lid-driven cavity flows. Comput. Fluids 100: 108-122

[4] Hassan M A and Jamal S 2014 Mixed convection in lid driven square cavity using finite volume method. Appl. Mech. Mater. 592-594: 1652-1656

[5] Ismael M A and Chamkha A J 2015 Mixed convection in liddriven trapezoidal cavities with an aiding or opposing side wall. Numer. Heat Transf. A 68: 312-335

[6] Ismael M A, Pop I and Chamkha A J 2014 Mixed convection in a lid-driven square cavity with partial slip. Int. J. Therm. Sci. 82: 47-61

[7] Khorasanizade S and Sousa J M M 2014 A detailed study of lid-driven cavity flow at moderate Reynolds numbers using Incompressible SPH. Int. J. Numer. Meth. Fluids 76(10): 653-668

[8] Roy M, Roy S and Basak T 2015 Role of various moving walls on energy transfer rates via heat flow visualization during mixed convection in square cavities. Energy 82: 1-22

[9] Sidik N A C and Razali S A 2014 Two-sided lid-driven cavity flow at different speed ratio by Lattice Boltzmann method. Appl. Mech. Math. 554: 675-679

[10] Sivakumar V and Sivasankaran S 2014 Mixed convection in an inclined lid-driven cavity with non-uniform heating on both sidewalls. J. Appl. Mech. Tech. Phys. 55(4): 634-649

[11] Sivasankaran S, Sivakumar V, Hussein A K and Prakash P 2014 Mixed convection in a lid-driven two dimensional square cavity with corner heating and internal heat generation. Numer. Heat Transf. A 65: 269-286

[12] Yapici K and Obut S 2015 Laminar mixed-convection heat transfer in a lid-driven cavity with modified heated wall. Heat Transf. Eng. 36(3): 303-314

[13] Barletta A and Nield D A 2009 Mixed convection with viscous dissipation and pressure work in a lid-driven square enclosure. Int. J. Heat Mass Transf. 52: 4244-4253

[14] Mahapatra S K, Nanda P and Sarkar A 2006 Interaction of mixed convection in two-sided lid driven differentially heated square enclosure with radiation in presence of participating medium. Heat Mass Transf. 42: 739-757

[15] Oztop H F and Dagtekin I 2004 Mixed convection in twosided lid-driven differentially heated square cavity. Int. J. Heat Mass Transf. 47: 1761-1769

[16] Wahba E M and Gadalla M A 2009 Heat and fluid flow characteristics inside differentially heated square enclosures with single and multiple sliding walls. Heat Trans. Asian Res. 38: 422-434

[17] Chatterjee D 2013 MHD mixed convection in a lid-driven cavity including a heated Source. Numer. Heat Transf. A, 64: 235-254

[18] Guo G and Sharif M A R 2004 Mixed convection in rectangular cavities at various aspect ratios with moving isothermal sidewalls and constant flux heat source on the bottom wall. Int. J. Therm. Sci. 43: 465-475

[19] Ramakrishna D, Basak T, Roy S and Pop I 2012 Numerical study of mixed convection within porous square cavities using Bejan's heatlines: effects of thermal aspect ratio and thermal boundary conditions. Int. J. Heat Mass Transf. 55: $5436-5448$

[20] Tiwari R K and Das M K 2007 Heat transfer augmentation in a two-sided lid-driven differentially heated square cavity utilizing nanofluids. Int. J. Heat Mass Transf. 50: 2002-2018

[21] Islam A W, Sharif M A R and Carlson E S 2012 Mixed convection in a lid driven square cavity with an isothermally heated square blockage inside. Int. J. Heat Mass Transf. 55: 5244-5255

[22] Morshed K N, Sharif M A R and Islam A W 2015 Laminar mixed convection in a lid driven square cavity with two isothermally heated square internal blockages. Chem. Eng. Commun. 202: 1176-1190

[23] Oztop H F, Zhao Z and Yu B 2009 Fluid flow due to combined convection in lid-driven enclosure having a circular body. Int. J. Heat Fluid Flow 30: 886-901

[24] Shankar P and Deshpande M 2000 Fluid mechanics in the driven cavity, Annu. Rev. Fluid Mech. 32(1): 93-136

[25] Schreiber R and Keller H B 1983 Driven cavity flows by efficient numerical techniques. J. Comput. Phys. 49: 310-333

[26] Basak T, Roy S, Sharma P K and Pop I 2009 Analysis of mixed convection flows within a square cavity with uniform and non-uniform heating of bottom wall. Int. J. Therm. Sci. 48: 891-912

[27] Aydin O and Yang W-J 2000 Mixed convection in cavities with a locally heated lower wall and moving sidewalls. Numer. Heat Transf. A 37: 695-710

[28] Bhattacharya M, Basak T, Oztop H F and Varol Y 2013 Mixed convection and role of multiple solutions in lid-driven trapezoidal enclosures. Int. J. Heat Mass Transf. 63: 366-388

[29] Moallemi M K and Jang K S 1992 Prandtl number effects on laminar mixed convection heat-transfer in a lid-driven cavity. Int. J. Heat Mass Transf. 35: 1881-1892

[30] Oztop H F, Sun C and Yu B 2008 Conjugate-mixed convection heat transfer in a lid-driven enclosure with thick bottom wall. Int. Commun. Heat Mass Transf. 35: 779-785

[31] Waheed M A 2009 Mixed convective heat transfer in rectangular enclosures driven by a continuously moving horizontal plate. Int. J. Heat Mass Transf. 52: 5055-5063

[32] Seyyedi S M, Soleimani S, Ghasemi E, Ganji D D, GorjiBandpy M and Bararnia H 2013 Numerical investigation of laminar mixed convection in a cubic cavity by MRT-LBM: effects of the sliding direction. Numer. Heat Transf. A 63: 285-304

[33] Ji T H, Kim S Y and Hyun J M 2007 Transient mixed convection in an enclosure driven by a sliding lid. Heat Mass Transf. 43: 629-638

[34] Sharif M A R 2007 Laminar mixed convection in shallow inclined driven cavities with hot moving lid on top and cooled from bottom. Appl. Therm. Eng. 27: 1036-1042

[35] Izadia M, Behzadmehrb A and Shahmardana M M 2015 Effects of inclination angle $\mathrm{n}$ mixed convection heat transfer of a nanofluid in a square cavity. Int. J. Comput. Meth. Eng. Sci. Mech. 16: 11-21

[36] Nada E A 2015 Dissipative particle dynamics simulation of combined convection in a vertical lid driven cavity with a corner heater. Int. J. Therm. Sci. 92: 72-84

[37] Oztop H F, Al-Salem K and Pop I 2011 MHD mixed convection in a lid-driven cavity with corner heater. Int. J. Heat Mass Transf. 54: 3494-3504 
[38] Sivakumar V, Sivasankaran S, Prakash P and Lee J 2010 Effect of heating location and size on mixed convection in lid-driven cavities. Comput. Math. Appl. 59: 3053-3065

[39] Muthtamilselvan M and Doh D H 2014 Mixed convection of heat generating nanofluid in a lid-driven cavity with uniform and non-uniform heating of bottom wall. Appl. Math. Mod. 38: $3164-3174$

[40] Kimura S and Bejan A 1983 The heatline visualization of convective heat transfer. J. Heat Transf. 105(4): 916-919

[41] Costa V A F 2003 Unified streamline, heatline and massline methods for the visualization of two-dimensional heat and mass transfer in anisotropic media. Int. J. Heat Mass Transf. 46: $1309-1320$

[42] Pilkington L A B 1969 Review lecture: the float glass process, Proc. R. Soc. Lond. A 314: 1-25

[43] Vishnuvardhanarao E and Das M K 2007 Laminar mixed convection in a parallel two-sided lid-driven differentially heated square cavity filled with a fluid-saturated porous medium. Numer. Heat Transf. A 53: 88-110

[44] Attia H A, Abbas W, Abdeen M A M and Said A A M 2015 Heat transfer between two parallel porous plates for Couette flow under pressure gradient and Hall current. Sadhan 40(1): 183-197

[45] Alloui Z and Vasseur P 2013 Fully developed mixed convection of a binary fluid in a vertical porous channel. Can. J. Chem. Eng. 91: 127-135

[46] Duwairi H M and Damseh R A 2004 MHD-buoyancy aiding and opposing flows with viscous dissipation effects from radiate vertical surfaces. Can. J. Chem. Eng. 82: 613-618

[47] Khan W A, Culham R and Makinde O D 2015 Hydromagnetic Blasius flow of power-law nanofluids over a convectively heated vertical plate. Can. J. Chem. Eng. 9999: 1-8

[48] Mansour M A and Ahmed S E 2012 Mixed convection flows in a square lid-driven cavity with heat source at the bottom utilising nanofluid. Can. J. Chem. Eng. 90: 100-110

[49] Prakash O M, Makinde O D, Kumar D and Dwivedi Y K 2015 Heat transfer to MHD oscillatory dusty fluid flow in a channel filled with a porous medium. Sadhana 40(4): 1273-1282

[50] Rahman M M and Al-Lawatia M 2010 Effects of higher order chemical reaction onmicropolar fluid flow on a power law permeable stretched sheet with variable concentration in a porous medium. Can. J. Chem. Eng. 88: 23-32

[51] Singh J, Choudhary N and Nigam K D P 2014 The thermal and transport characteristics of nanofluids in a novel three dimensional device. Can. J. Chem. Eng. 92: 2185-2201

[52] Subramanian S, Swain P K, Deshpande A V and Satyamurthy P 2015 Effect of Hartmann layer resolution for MHD flow in a straight, conducting duct at high Hartmann numbers. Sadhana 40(3): 851-861

[53] Bejan A 2004 Convection heat transfer, 3rd edition, Hoboken: Wiley

[54] Bansal S and Chatterjee D 2015 Magneto-convective transport of nanofluid in a vertical lid-driven cavity including a heat-conducting rotating circular cylinder. Numer. Heat Transf. A 68: 411-431

[55] Mukhopadhyay A 2010 Analysis of entropy generation due to natural convection in square enclosures with multiple discrete heat sources. Int. Commun. Heat Mass Transf. 37: 867-872

[56] Deng Q H and Tang G F 2002 Numerical visualization of mass and heat transport for mixed convective heat transfer by streamline and heatline. Int. J. Heat Mass Transf. 45: 2387-2396

[57] Hooman K 2010 Energy flux vector as a new tool for convection visualization. Int. J. Numer. Methods Heat Fluid Flow 20: 240-249

[58] Patankar S V 1980 Numerical heat transfer and fluid flow. Milton Park: Taylor \& Francis

[59] Biswas N, Mahapatra P S and Manna N K 2015 Mixed convection heat transfer in a grooved channel. Numer. Heat Transf. A 68: 663-685

[60] Sharma A K, Mahapatra P S, Manna N K and Ghosh K 2015 Mixed convection in a baffled grooved channel. Sadhana 40(3): 835-849

[61] Abdelkhalek M M 2008 Mixed convection in a square cavity by a perturbation technique. Comput. Mater. Sci. 42: 212-219

[62] Iwatsu E M 2009 Multiplicity of states for two-sided and four-sided lid driven cavity flows. Comput. Fluids 38: 247-253 